Pacific

Journal of

Mathematics

\title{
CYCLIC BRANCHED COVERINGS OF KNOTS AND QUANDLE HOMOLOGY
}

\author{
YUICHI KABAYA
}




\title{
CYCLIC BRANCHED COVERINGS OF KNOTS AND QUANDLE HOMOLOGY
}

\author{
YUICHI KABAYA
}

\begin{abstract}
We give a construction of quandle cocycles from group cocycles, especially, for any integer $p \geq 3$, quandle cocycles of the dihedral quandle $R_{p}$ from group cocycles of the cyclic group $\mathbb{Z} / p$. We show that a group 3-cocycle of $\mathbb{Z} / p$ gives rise to a nontrivial quandle 3-cocycle of $R_{p}$. When $p$ is an odd prime, since $\operatorname{dim}_{\mathbb{F}_{p}} H_{Q}^{3}\left(R_{p} ; \mathbb{F}_{p}\right)=1$, our 3-cocycle is a constant multiple of the Mochizuki 3-cocycle up to coboundary. Dually, we construct a group cycle represented by a cyclic branched covering branched along a knot $K$ from the quandle cycle associated with a colored diagram of $K$.
\end{abstract}

\section{Introduction}

A quandle, which was introduced by Joyce [1982], is an algebraic object whose axioms are motivated by knot theory and conjugation in a group. Carter, Jelsovsky, Kamada, Langford and Saito [Carter et al. 2003] introduced a quandle homology theory, and they defined the quandle cocycle invariants for classical knots and surface knots. The quandle homology is defined as the homology of a chain complex generated by cubes whose edges are labeled by elements of a quandle. On the other hand, the group homology is defined as the homology of a chain complex generated by tetrahedra whose edges are labeled by elements of a group. So it is natural to ask for a relation between quandle homology and group homology. This question also arises from the fact that the quandle cocycle invariants were defined as an analogue of the Dijkgraaf-Witten invariants, which are defined using group cocycles.

In [Inoue and Kabaya 2010], we defined a simplicial version of quandle homology and constructed a homomorphism from the usual quandle homology to the simplicial quandle homology. The important point is that this homomorphism gives a triangulation of a knot complement in algebraic fashion. This enables us to relate the quandle homology to the topology of knot complements.

The author is supported by JSPS Research Fellowships for Young Scientists. MSC2010: 57M05, 57M10, 57M12, 57M25, 57M27.

Keywords: quandle homology, group homology, cyclic branched covering. 
In this paper, we apply the results of [Inoue and Kabaya 2010] to construct quandle cocycles from group cocycles. First, we demonstrate how to give a quandle cocycle of the dihedral quandle $R_{p}$ from a group cocycle of the cyclic group $\mathbb{Z} / p$ for any integer $p \geq 3$ in Section 7 . We show that a generator of $H^{3}(\mathbb{Z} / p ; \mathbb{Z} / p)$ gives rise to a nontrivial quandle 3 -cocycle of $H_{Q}^{3}\left(R_{p} ; \mathbb{Z} / p\right)$. When $p$ is an odd prime, since $\operatorname{dim}_{\mathbb{F}_{p}} H_{Q}^{3}\left(R_{p} ; \mathbb{F}_{p}\right)=1$, our quandle 3 -cocycle is equal to a constant multiple of the Mochizuki 3-cocycle [2003] up to coboundary.

Then we generalize the construction to wider classes of quandles. Let $G$ be a group and let $h$ be an element of $G$; then the set $\operatorname{Conj}(h)=\left\{g^{-1} h g \mid g \in G\right\}$ forms a quandle by conjugation. (It is known from [Joyce 1982] that any faithful homogeneous quandle has such a presentation.) When some obstruction in second cohomology vanishes, we construct a quandle cocycle of $\operatorname{Conj}(h)$ from a group cocycle of $G$.

Dually, we relate the quandle cycle associated with an arc and region coloring (shadow coloring) of a knot $K$ to a group cycle represented by a cyclic branched covering branched along $K$. Let $D$ be a diagram of $K$. We can define the notion of arc and region colorings of $D$ by a quandle $\operatorname{Conj}(h)$. A pair of an arc and a region coloring is called a shadow coloring. We can associate a cycle of a quandle homology group to a shadow coloring of $D$. Using the homomorphism constructed in [Inoue and Kabaya 2010], we construct a group cycle of $G$ represented by a cyclic branched covering branched along $K$. This reveals a close relationship between the shadow cocycle invariant of a knot and the Dijkgraaf-Witten invariant of the cyclic branched cover.

Hatakenaka and Nosaka [2012] defined an invariant of 3-manifolds called the 4-fold symmetric quandle homotopy invariant, based on the fact that any 3-manifold can be represented as a 4-fold simple branched covering of $S^{3}$ along a link. As an application, they showed that the shadow cocycle invariant of a link for the Mochizuki 3-cocycle is equal to a scalar multiple of the Dijkgraaf-Witten invariant of the double branched cover along the link.

This paper is organized as follows. In Section 2, we recall the definition of group homology and show how to represent a group cycle by a triangulation with a labeling of its 1-simplices. We give a presentation of the fundamental group of a cyclic branched covering branched along a knot in Section 3, which is independent from the other sections. In Section 4, we construct a group cycle represented by a cyclic branched cover. We recall the definition of quandles and their homology theory in Section 5. We review some results from [Inoue and Kabaya 2010] in Section 6 and apply them to construct quandle cocycles of the dihedral quandle $R_{p}$ in Section 7. The reader interested in the form of the 3-cocycle should consult (7-5) (and (7-4)). We generalize the construction to wider classes of quandles in Section 8. In Section 9, we construct a group cycle represented by a cyclic branched 
covering from the quandle cycle associated with a shadow coloring. The reader who is only interested in the construction of quandle cocycles from group cocycles may skip Sections 2B 2C 3, and 4,

\section{Group homology}

In this section, we collect basic facts on group homology, starting with a review of definitions; see [Brown 1982] for details. The material discussed in Sections 2B and $2 \mathrm{C}$ was developed in [Neumann 2004].

2A. Group homology. Let $G$ be a group. Let $C_{n}(G)$ be the free $\mathbb{Z}[G]$-module generated by $n$-tuples $\left[g_{1}|\ldots| g_{n}\right]$ of elements of $G$. Define the boundary map д : $C_{n}(G) \rightarrow C_{n-1}(G)$ by

$$
\begin{aligned}
& \partial\left(\left[g_{1}|\ldots| g_{n}\right]\right) \\
& \quad=g_{1}\left[g_{2}|\ldots| g_{n}\right]+\sum_{i=1}^{n-1}(-1)^{i}\left[g_{1}|\ldots| g_{i} g_{i+1}|\ldots| g_{n}\right]+(-1)^{n}\left[g_{1}|\ldots| g_{n-1}\right] .
\end{aligned}
$$

We remark that the chain complex $\left\{\cdots \rightarrow C_{1}(G) \rightarrow C_{0}(G) \rightarrow \mathbb{Z} \rightarrow 0\right\}$ is acyclic, where $C_{0}(G) \cong \mathbb{Z}[G] \rightarrow \mathbb{Z}$ is the augmentation map. So the chain complex $C_{*}(G)$ gives a free resolution of $\mathbb{Z}$. Let $M$ be a right $\mathbb{Z}[G]$-module. The homology of $C_{n}(G ; M)=M \otimes_{\mathbb{Z}[G]} C_{n}(G)$ is called the group homology of $M$ and denoted by $H_{n}(G ; M)$. In other words, $H_{n}(G ; M)=\operatorname{Tor}_{n}^{\mathbb{Z}[G]}(M, \mathbb{Z})$.

Let $C_{n}^{\prime}(G)$ be the free $\mathbb{Z}$-module generated by $\left(g_{0}, \ldots, g_{n}\right) \in G^{n+1}$. Then $C_{n}^{\prime}(G)$ is a left $\mathbb{Z}[G]$-module by the action $g\left(g_{0}, \ldots, g_{n}\right)=\left(g g_{0}, \ldots, g g_{n}\right)$. Define the boundary operator of $C_{n}^{\prime}(G)$ by

$$
\partial\left(g_{0}, \ldots, g_{n}\right)=\sum_{i=0}^{n}(-1)^{i}\left(g_{0}, \ldots, \widehat{g_{i}}, \ldots, g_{n}\right) .
$$

$C_{*}(G)$ and $C_{*}^{\prime}(G)$ are isomorphic as chain complexes. In fact, the following correspondence gives an isomorphism:

$$
\left[g_{1}\left|g_{2}\right| \ldots \mid g_{n}\right] \leftrightarrow\left(1, g_{1}, g_{1} g_{2}, \ldots, g_{1} \cdots g_{n}\right),
$$

or, equivalently,

$$
g_{0}\left[g_{0}^{-1} g_{1}\left|g_{1}^{-1} g_{2}\right| \ldots \mid g_{n-1}^{-1} g_{n}\right] \leftrightarrow\left(g_{0}, \ldots, g_{n}\right) .
$$

The notation that uses $\left(g_{0}, \ldots, g_{n}\right)$ is called homogeneous, and the one that uses $\left[g_{1}|\ldots| g_{n}\right]$ is called inhomogeneous.

Factoring out $C_{n}(G)$ by the degenerate subcomplex generated by $\left[g_{1}|\ldots| g_{n}\right]$ such that $g_{i}=1$ for some $i$, we obtain the normalized chain complex and its homology group. It is known that the group homology using the normalized 
chain complex coincides with the homology using the unnormalized one. In the homogeneous notation, we factor out $C_{n}^{\prime}(G)$ by the subcomplex generated by $\left(g_{0}, \ldots, g_{n}\right)$ such that $g_{i}=g_{i+1}$ for some $i$ to define the normalized chain complex.

For a left $\mathbb{Z}[G]$-module $N$, the group cohomology $H^{n}(G ; N)$ is defined as the cohomology of the cochain complex $C^{n}(G ; N)=\operatorname{Hom}_{\mathbb{Z}[G]}\left(C_{n}(G), N\right)$. Let $A$ be an abelian group. A cocycle of $C^{n}(G ; A)$ in the homogeneous notation is a function $f: G^{n+1} \rightarrow A$ satisfying the following conditions:

(1) $\sum_{i=0}^{n+1}(-1)^{i} f\left(x_{0}, \ldots, \widehat{x_{i}}, \ldots, x_{n+1}\right)=0$,

(2) $f\left(g x_{0}, \ldots, g x_{n}\right)=f\left(x_{0}, \ldots, x_{n}\right)$ for any $g \in G$ (left invariance).

If $f$ also satisfies

(3) $f\left(x_{0}, \ldots, x_{n}\right)=0$ if $x_{i}=x_{i+1}$ for some $i$,

then $f$ is a normalized $n$-cocycle. We can show that any $n$-cocycle is cohomologous to a normalized $n$-cocycle.

2B. Cycles represented by triangulations. Let $\Delta$ be an $n$-dimensional simplex. We label the vertices of $\Delta$ by $0,1, \ldots, n$. A face of $\Delta$ is presented by a subset of $\{0,1, \ldots, n\}$. Let $\left\langle i_{0}, \ldots, i_{k}\right\rangle$ be the face spanned by $i_{0}, \ldots, i_{k} \in\{0, \ldots, n\}$, with a vertex ordering given by $i_{0}, \ldots, i_{k}$. Any face inherits a vertex ordering from the vertex ordering of $\Delta$, that is, $\left\langle i_{0}, \ldots i_{k}\right\rangle$ with $i_{0}<i_{1}<\cdots<i_{k}$.

Definition 2.1. Let $T$ be a CW-complex obtained by gluing a finite number of $n$-dimensional simplices along their $(n-1)$-dimensional faces in pairs by simplicial homeomorphisms. We denote the $k$-skeleton of $T$ by $T^{(k)}$. We assume that the gluing maps preserve the vertex orderings of the faces. Then $T-T^{(n-3)}$ is homeomorphic to a topological $n$-manifold (not orientable in general). When $T-T^{(n-3)}$ is oriented, we call $T$ an ordered $n$-cycle.

Consider an $n$-cycle $\sigma$ of $C_{n}(G ; \mathbb{Z})$. Then $\sigma$ is represented by a sum

$$
\sum_{j} \epsilon_{j}\left[g_{j 1}|\ldots| g_{j n}\right]
$$

where $\epsilon_{j}= \pm 1$ and $g_{j k} \in G$. For each $\left[g_{j 1}|\ldots| g_{j n}\right]$, take an $n$-simplex $\Delta_{j}$. Then label the edge $\left\langle i_{1} i_{2}\right\rangle$ of $\Delta_{j}$ by $g_{j i_{1}} g_{j\left(i_{1}+1\right)} \ldots g_{j i_{2}}$ for $i_{1}<i_{2}$. In particular, we have

$$
\langle 0,1\rangle \leftrightarrow g_{j 1}, \quad\langle 1,2\rangle \leftrightarrow g_{j 2}, \quad \ldots, \quad\langle n-1, n\rangle \leftrightarrow g_{j n} .
$$

We denote the label of $\left\langle i_{1}, i_{2}\right\rangle$ by $\lambda\left\langle i_{1}, i_{2}\right\rangle$. For $i_{1}>i_{2}$, label the oriented edge $\left\langle i_{1}, i_{2}\right\rangle$ by $\lambda\left\langle i_{2}, i_{1}\right\rangle^{-1}$. For any 2 -dimensional face $\left\langle i_{0}, i_{1}, i_{2}\right\rangle$, we have

$$
\lambda\left\langle i_{0}, i_{1}\right\rangle \lambda\left\langle i_{1}, i_{2}\right\rangle=\lambda\left\langle i_{0}, i_{2}\right\rangle .
$$




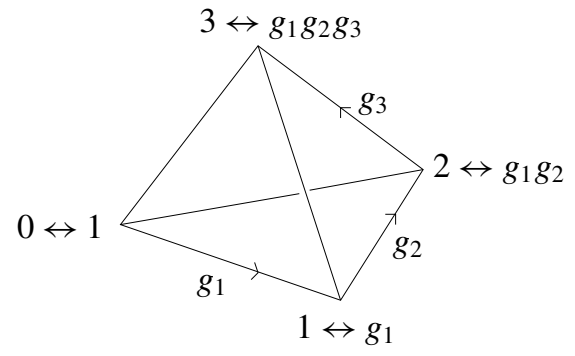

Figure 1. A labeling of a simplex.

Rewriting them in the homogeneous notation, we assign labels to the vertices of $\Delta_{j}$ as

$$
0 \leftrightarrow 1, \quad 1 \leftrightarrow g_{j 1}, \quad 2 \leftrightarrow g_{j 1} g_{j 2}, \quad \ldots, \quad n \leftrightarrow g_{j 1} \ldots g_{j n},
$$

up to the left action of $G$ (Figure 1).

Since $\partial \sigma=0,(n-1)$-dimensional faces cancel in pairs. Gluing the $\Delta_{j}$ 's along their faces according to such pairings, we obtain an $n$-cycle $T$. At any $(n-1)$ simplex of $T$, there exist exactly two adjacent $n$-simplices. The labelings of the $(n-1)$-simplex derived from these two $n$-simplices coincide. Thus we have a welldefined labeling of 1-simplices $\lambda$ : \{oriented 1-simplices of $T\} \rightarrow G$ satisfying:

(1) $\lambda\left\langle i_{0}, i_{1}\right\rangle \lambda\left\langle i_{1}, i_{2}\right\rangle=\lambda\left\langle i_{0}, i_{2}\right\rangle$ for any 2 -dimensional face $\left\langle i_{0}, i_{1}, i_{2}\right\rangle$,

(2) $\lambda\left\langle i_{1}, i_{0}\right\rangle=\lambda\left\langle i_{0}, i_{1}\right\rangle^{-1}$.

We call a labeling of 1-simplices satisfying the conditions (1) and (2) a $G$-valued 1-cocycle. Orient $\Delta_{j}$ positively if $\epsilon_{j}=1$ and negatively if $\epsilon_{j}=-1$. Since these orientations agree on face pairings, we thus have an orientation on $T$. Therefore $T$ is an ordered $n$-cycle with a $G$-valued 1-cocycle $\lambda$. In general, $T$ may not be connected, but we assume that $T$ is connected because we can treat each connected component separately in our arguments. Conversely, any ordered $n$-cycle $T$ with a $G$-valued 1-cocycle $\lambda$ represents an $n$-cycle of $C_{n}(G ; \mathbb{Z})$.

2C. Group cycles and representations. Suppose a cycle $\sigma \in C_{n}(G ; \mathbb{Z})$ is represented by an ordered $n$-cycle $T$ with a $G$-valued 1-cocycle $\lambda$. Then $\sigma$ induces a homomorphism from $\pi_{1}(T)$ to $G$ as follows. Let $\widetilde{T}$ be the universal covering of $T$ and let $p: \widetilde{T} \rightarrow T$ be the covering map. Then the simplices of $T$ lift to simplices of $\widetilde{T}$, and each lift has an induced vertex ordering compatible with adjacent $n$-simplices. The $G$-valued 1-cocycle $\lambda$ of $T$ induces a $G$-valued 1-cocycle of $\widetilde{T}$. Consider a fundamental domain of $T$, that is, a contractible subcomplex $D$ of $\widetilde{T}$ such that

- $\widetilde{T}=\bigcup_{\gamma \in \pi_{1}(M)} \gamma D$

- $D \cap \gamma D=$ (lower-dimensional simplices), for any $\gamma \neq 1$, 
where we regard $\pi_{1}(T)$ as the group of deck transformations. By definition, the number of $n$-simplices in $D$ coincides with the number of $n$-simplices in $T$; in particular, both are finite. We fix a base point $\widetilde{*}$ in the interior of $D$. Each $(n-1)$ simplex on $\partial D$ is glued to another $(n-1)$-simplex on $\partial D$. We denote such a pair of faces by $F_{i}^{ \pm}$. Let $x_{i}$ be a path in $T$ that starts at $*=p(\widetilde{*})$, traverses $p\left(F_{i}\right)$ in the direction from $F_{i}^{+}$to $F_{i}^{-}$, and ends at $*$. These paths form a system of generators of the fundamental group $\pi_{1}(T, *)$. The relations are given at any $(n-2)$-simplices, around which there are a finite number of $p\left(F_{i}\right)$ 's.

Fix an $n$-simplex $\Delta$ in $D$ and a labeling of vertices of $\Delta$ derived from $\lambda$. Then $n$-simplices adjacent to $\Delta$ inherit labelings of vertices from $\lambda$. In this way, all vertices of $D$ are labeled by elements of $G$. Now consider the labeling of vertices of $F_{i}^{+}$and $F_{i}^{-}$. Since these reduce to the same labeling of edges, they coincide up to left multiplication. Therefore there exists an element of $G$ that sends the labeling of vertices of $F_{i}^{-}$to the one of $F_{i}^{+}$. Denote the element by $\rho\left(x_{i}\right)$. This $\rho$ induces a homomorphism $\rho: \pi_{1}(T, *) \rightarrow G$.

Conversely, if we have an ordered $n$-cycle $T$ and a homomorphism

$$
\rho: \pi_{1}(T, *) \rightarrow G
$$

we can construct a $G$-valued 1 -cocycle $\lambda$ and then a cycle of $C_{n}(G ; \mathbb{Z})$ up to boundary as follows. Since $\rho$ induces a map

$$
T \rightarrow K\left(\pi_{1}(T, *), 1\right) \rightarrow B G
$$

we obtain a labeling of 1 -simplices $\lambda$ of $T$. This gives rise to a $G$-valued 1 -cocycle $\lambda$ and a homology class in $H_{n}(G ; \mathbb{Z})$. The $G$-valued 1-cocycle $\lambda$ is well-defined up to the coboundary action. A map $\mu:\{0$-simplices of $T\} \rightarrow G$ acts on a $G$-valued 1 -cocycle $\lambda$ as a coboundary action by

$$
\left\langle i_{1}, i_{2}\right\rangle \mapsto \mu\left(i_{1}\right)^{-1} \lambda\left\langle i_{1}, i_{2}\right\rangle \mu\left(i_{2}\right) .
$$

We can show that the homology class obtained from $\lambda$ does not change under the coboundary action. For any $g \in G$, the cycle corresponding to the representation $g^{-1} \rho g$ is obtained from $\lambda$ by the coboundary action by $\mu \equiv g$. As a result, the homology class obtained from $\rho$ depends only on the conjugacy class of $\rho$.

For a closed oriented $n$-manifold $M$ and a representation $\rho: \pi_{1}(M) \rightarrow G$, we have a homology class defined by the image of the fundamental class $[M]$ under the map $H_{n}(M) \rightarrow H_{n}\left(K\left(\pi_{1}(M), 1\right)\right) \rightarrow H_{n}(G ; \mathbb{Z})$. When $M$ is homeomorphic to an ordered $n$-cycle $T$, the homology class is represented by a $G$-valued 1-cocycle $\lambda$ of $T$ associated with $\rho$. In this situation, we say that the homology class defined by $M$ and $\rho$ is represented by $T$ and $\lambda$. 

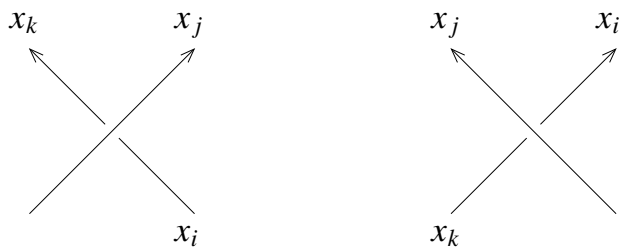

Figure 2. The relation is given by $x_{k}=x_{j}^{-1} x_{i} x_{j}$.

\section{Cyclic branched covering}

In this section, we give a presentation of the fundamental group of a cyclic branched covering from the Wirtinger presentation.

3A. Presentation of the fundamental group of the branched cover. Let $K$ be a knot in $S^{3}$ and let $D$ be a diagram of $K$. Then $\pi_{1}\left(S^{3}-K\right)$ is presented by generators and relations called the Wirtinger presentation. Let $x_{1}, \ldots, x_{n}$ be the generators of the Wirtinger presentation that correspond to the $\operatorname{arcs}$ of $D$. Each crossing (Figure 2) gives rise to a relation $x_{k}=x_{j}^{-1} x_{i} x_{j}$.

For any integer $l>1$, let $C_{l}$ be the $l$-fold cyclic covering of $K$, that is, the manifold corresponding to the kernel of

$$
\pi_{1}\left(S^{3}-K\right) \rightarrow H_{1}\left(S^{3}-K\right) \cong \mathbb{Z} \rightarrow \mathbb{Z} / l .
$$

Putting back the knot $K$ to $C_{l}$, we obtain the $l$-fold cyclic branched covering $\widehat{C}_{l}$ of $K$.

Proposition 3.1. $\pi_{1}\left(C_{l}\right)$ has the following presentation:

Generators: $x_{i, s}($ for $i=1,2, \ldots, n$ and $s=0,1, \ldots, l-1)$,

Relations: $x_{k, s}=x_{j, s-1}^{-1} x_{i, s-1} x_{j, s}($ for each crossing and $s=0,1, \ldots, l-1)$,

$$
x_{1,0}=x_{1,1}=\cdots=x_{1, l-2}=1 .
$$

The inclusion map $\pi_{1}\left(C_{l}\right) \rightarrow \pi_{1}\left(S^{3}-K\right)$ is given by

$$
x_{i, s} \mapsto x_{1}^{s-1} x_{i} x_{1}^{-s},
$$

if we take appropriate base points. By adding a relation $x_{1, l-1}=1$, we obtain a presentation of $\pi_{1}\left(\widehat{C}_{l}\right)$.

A method for obtaining a presentation of the fundamental group of a branched covering is given in [Rolfsen 1976]. But we give a proof here because some techniques are used to construct a group cycle represented by $\widehat{C}_{l}$ later.

Proof. First, we construct a handle decomposition of the knot complement associated with the Wirtinger presentation (Figure 3). Then we lift the handle decomposition 


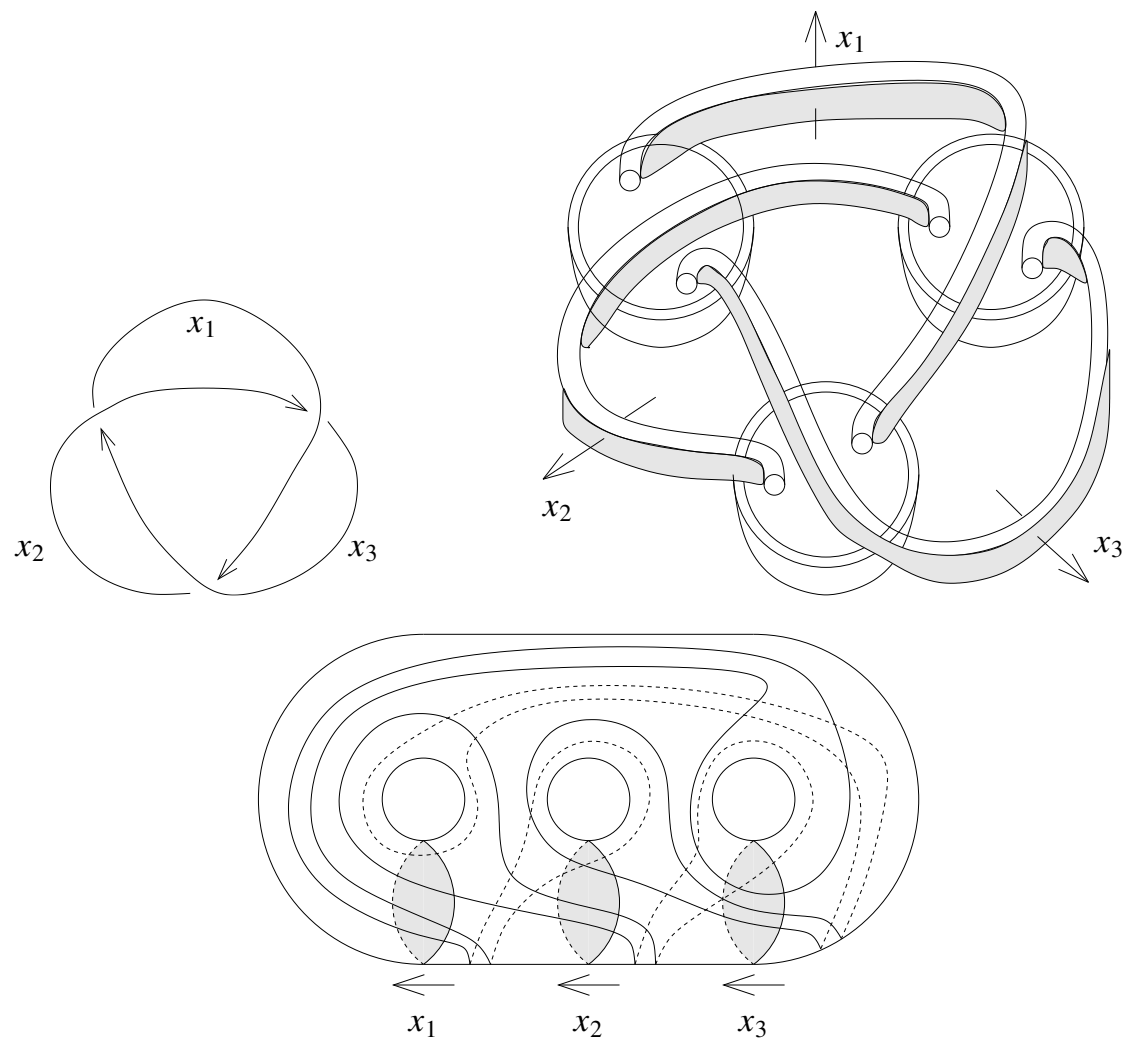

Figure 3. A handle decomposition of a knot complement.

to a handle decomposition of $C_{l}$ (Figure 4). After that, we read the relations given by attaching 2-handles.

Let $N(K)$ be a regular neighborhood of $K$. We give a handle decomposition of $S^{3}-N(K)$. We represent $S^{3}$ by the one-point compactification of

$$
\mathbb{R}^{3}=\{(x, y, z) \mid x, y, z \in \mathbb{R}\} .
$$

Let $B_{+}=\{z \geq 0\} \cup\{\infty\}$ and $B_{-}=\{z \leq 0\} \cup\{\infty\}$. We denote the equatorial sphere of $S^{3}$ by $S_{0}=\{z=0\} \cup\{\infty\}$. Put $K$ in a position such that the projection to $S_{0}$ has only double points. Let $n$ be the number of crossings of this projection. We deform $K$ in the $z$-direction so that $K$ intersects $S_{0}$ at $2 n$ points and each of $B_{ \pm} \cap K$ consists of $n$ arcs. We call $B_{+} \cap K$ over-crossing arcs and $B_{-} \cap K$ under-crossing arcs. Index the arcs of $B_{+} \cap K$ by $x_{i}(i=1,2, \ldots, n)$.

Now $B_{+}-N(K)$ is homeomorphic to a handlebody of genus $n$ (Figure 3). Projecting the over-crossing arcs to $S_{0}$, we obtain a meridian disk system of the handlebody. We denote the meridian disk corresponding to $x_{i}$ by $D_{i}$. For each under-crossing arc, attach a 2-handle $D^{2} \times D^{1}$ along $\partial D^{2} \times D^{1}$ to $B_{+}-N(K)$ 
(Figure 3). Then the resulting manifold is homeomorphic to $\left(S^{3}-N(K)\right)-B^{3}$, where $B^{3}$ is a 3-ball. Attaching $B^{3}$ along the boundary to $\partial\left(\left(S^{3}-N(K)\right)-B^{3}\right)$, the resulting manifold is homeomorphic to $S^{3}-N(K)$. So we have a handle decomposition of $S^{3}-N(K)$ into one 0-handle, $n$ 1-handles, $n$ 2-handles and one 3-handle. In this handle decomposition, 1-handles correspond to the Wirtinger generators and 2-handles to the Wirtinger relations. We denote the set of $i$-handles by $h^{i}$ and $X^{(i)}=h^{0} \cup \cdots \cup h^{i}$.

Next we consider the preimage of $X^{(1)}$ in $C_{l}$. Cut $X^{(1)}$ along the meridian disks $D_{i}$, and denote the resulting manifold by $B$, which is homeomorphic to a 3-ball. Let $*$ be a point in $B \subset S^{3}-N(K)$. We take a loop in $X^{(1)}$ that starts at $*$, intersects the meridian disk $D_{i}$, and ends at $*$. Orient the loop so that it corresponds to the generator of $H_{1}\left(S^{3}-K\right)$. By abuse of notation, we also denote this loop by $x_{i}$. Take a lift $B_{0} \subset C_{l}$ of $B$ and denote the preimage of $* \in B$ in $B_{0}$ by $\tilde{*}$. Then there exists a unique lift $\tilde{x}_{i}$ of the loop $x_{i}$ starting at $\tilde{*}$. Since $x_{i}$ corresponds to the generator of $H_{1}\left(S^{3}-K\right), \tilde{x}_{i}$ ends at another lift of $B$, which we denote by $B_{1}$. Similarly, the lift of $x_{i}$ starting at $B_{1}$ ends at another lift, which we denote by $B_{2}$. Continuing this, we see that $B_{l}=B_{0}$ and all lifts of $B$ will appear. Therefore the preimage of $X^{(1)}$ is decomposed into $l$ 3-balls $B_{0} \cup B_{1} \cup \cdots \cup B_{l-1}$. The intersection of $B_{s}$ and $B_{s+1}$ consists of $n$ disks, each of which is a lift of a meridian disk $D_{i}$. We denote this lifted disk by $D_{i, s}$ (Figure 4 ). It is easy to check that

$$
\left\{D_{1, l-1}\right\} \cup\left\{D_{i, s}: i=2, \ldots, n ; s=0, \ldots, l-1\right\}
$$

forms a meridian disk system of the preimage of $X^{(1)}$. Denote by $\tilde{x}_{i, s}$ the lift of $x_{i}$ starting at $B_{s}$ and ending at $B_{s+1}$.

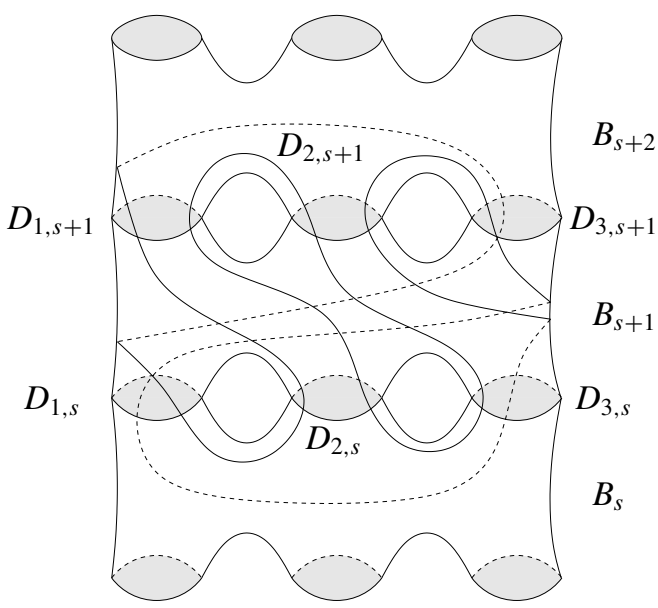

Figure 4. A handle decomposition of $C_{l}$. 
The generator of $\pi_{1}\left(C_{l}, \widetilde{*}\right)$ corresponding to the meridian disk $D_{i, s}$ is given by

$$
\tilde{x}_{1,0} \tilde{x}_{1,1} \ldots \tilde{x}_{1, s-1} \tilde{x}_{i, s} \tilde{x}_{1, s}^{-1} \tilde{x}_{1, s-1}^{-1} \ldots \tilde{x}_{1,0}^{-1} .
$$

We denote this element by $x_{i, s}$. To give a simple presentation of $\pi_{1}\left(C_{l}, \widetilde{*}\right)$, we add extra generators $x_{1,0}, x_{1,1}, \ldots, x_{1, l-2}$ corresponding to $\tilde{x}_{1,0}, \tilde{x}_{1,1}, \ldots \tilde{x}_{1, l-2}$, respectively, and relations $x_{1,0}=x_{1,1}=\cdots=x_{1, l-2}=1$.

Finally, we consider the relations given by the lifts of 2-handles. We see that the relation $x_{k}=x_{j}^{-1} x_{i} x_{j}$ lifts to

$$
x_{k, s}=x_{j, s-1}^{-1} x_{i, s-1} x_{j, s} \quad(s=0,1, \ldots, l-1)
$$

see Figure 4.

Since the generator $x_{i, s}$ is represented by (3-1), the inclusion map $\pi_{1}\left(C_{l}, \tilde{*}\right) \rightarrow$ $\pi_{1}\left(S^{3}-K, *\right)$ is given by $x_{i, s} \mapsto x_{1}^{s-1} x_{s} x_{1}^{-s}$. This proves the second statement.

By adding a 2-handle to $C_{l}$ along $\tilde{x}_{1,0} \tilde{x}_{1,1} \ldots \tilde{x}_{1, l-1}$ and capping off the resulting sphere, we obtain a manifold homeomorphic to the cyclic branched covering $\widehat{C}_{l}$. Therefore a presentation of the cyclic branched covering is obtained by adding a relation $x_{1, l-1}=1$.

\section{Cycle represented by cyclic branched covering}

For a representation $\rho: \pi_{1}\left(S^{3}-K\right) \rightarrow G$, we have the restriction map $\left.\rho\right|_{\pi_{1}\left(C_{l}\right)}$ : $\pi_{1}\left(C_{l}\right) \rightarrow G$ given by

$$
\left.\rho\right|_{\pi_{1}\left(C_{l}\right)}\left(x_{i, s}\right)=\rho\left(x_{1}\right)^{s-1} \rho\left(x_{i}\right) \rho\left(x_{1}\right)^{-s} .
$$

If $\rho\left(x_{1}\right)^{l}=1$, it reduces to a representation $\widehat{\rho}: \pi_{1}\left(\widehat{C}_{l}\right) \rightarrow G$ and there is a group cycle given by $\widehat{C}_{l}$ and $\widehat{\rho}$. In this section, we construct an explicit ordered 3-cycle and its $G$-valued 1 -cocycle representing the homology class given by $\widehat{C}_{l}$ and $\widehat{\rho}$. First we give a triangulation of $S^{3}-N(K)$ associated with the Wirtinger presentation.

Let $K$ be a knot and fix a diagram of $K$. As in the proof of Proposition 3.1, we define $B_{ \pm}$and give a Heegaard splitting of $S^{3}-N(K)$ with the meridian disks $D_{i}$. Cutting the handlebody $B_{+}-N(K)$ along the meridian disks $D_{i}$, the result is a ball with $2 n 2$-cells on the boundary. We denote the resulting 3-ball by $B$ and the pair of 2-cells corresponding to $D_{i}$ by $F_{i}^{+}$and $F_{i}^{-}$so that the Wirtinger generator corresponding to $x_{i}$ runs from $F_{i}^{+}$to $F_{i}^{-}$(Figure 5(A)). Now consider the attaching regions of the two handles corresponding to under-crossing arcs, which consist of annuli $S^{1} \times D^{1}\left(\subset D^{2} \times D^{1}\right)$ on $\partial\left(B_{+}-N(K)\right)$. Each annulus is divided by the $D_{i}$ 's into four rectangles on $\partial B$. We define a graph on $\partial B$ with vertices consisting of $F_{i}^{ \pm}$and edges consisting of these rectangles (Figure 5(B)). Each vertex of this graph has valency at least four. We can make all vertices of the graph into trivalent vertices (Figure 5(C)) by adding extra 1-handles and 2-handles (stabilizations of 

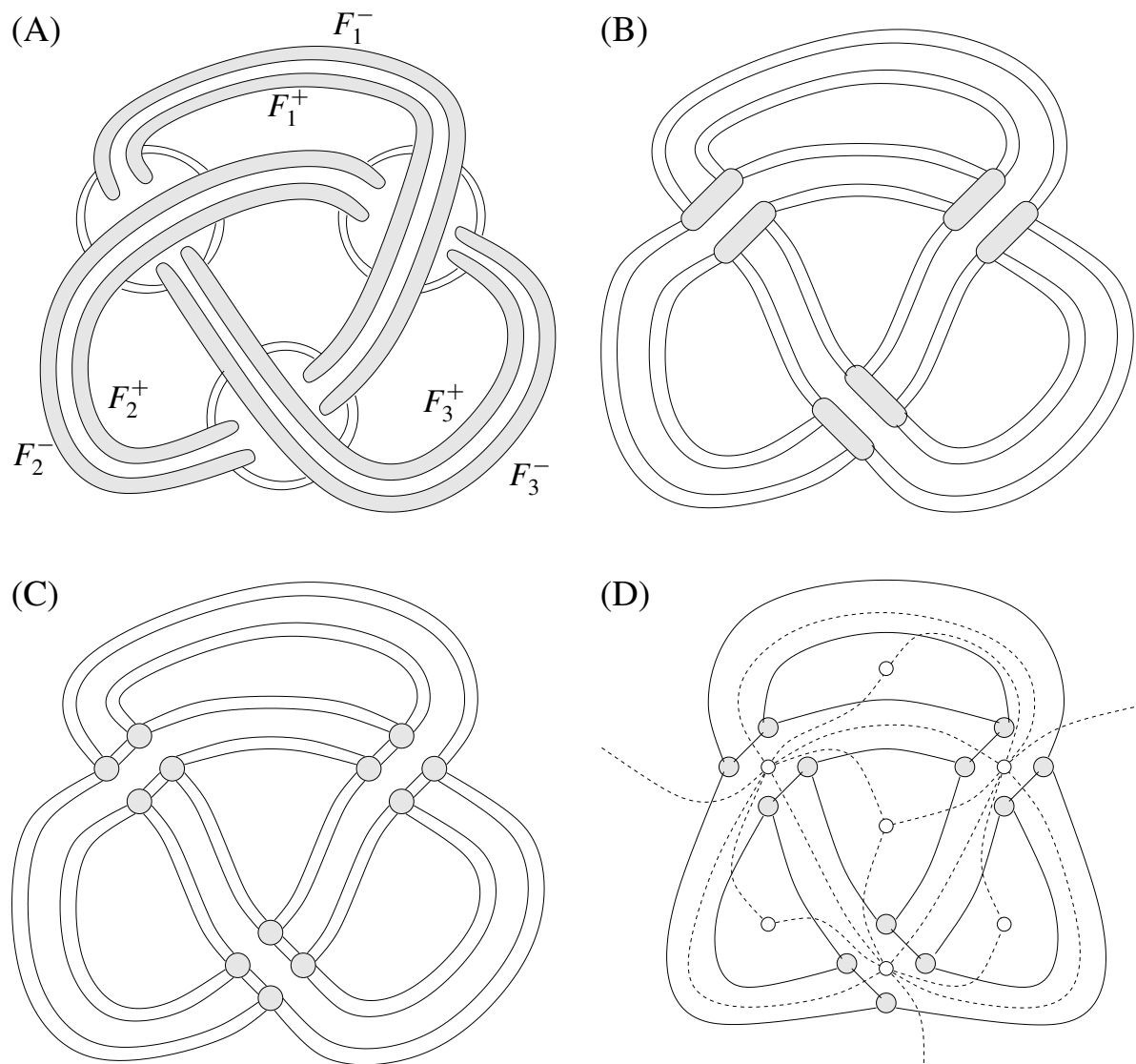

(D)

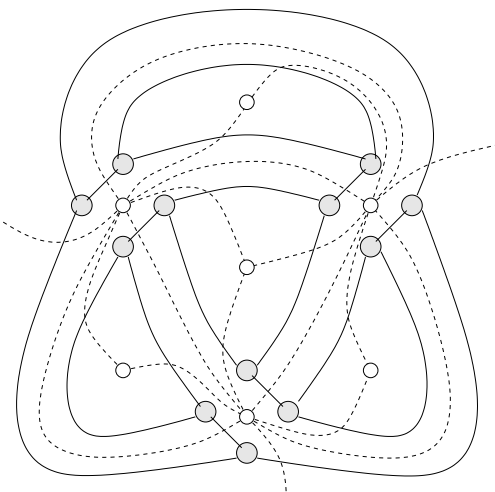

(E)

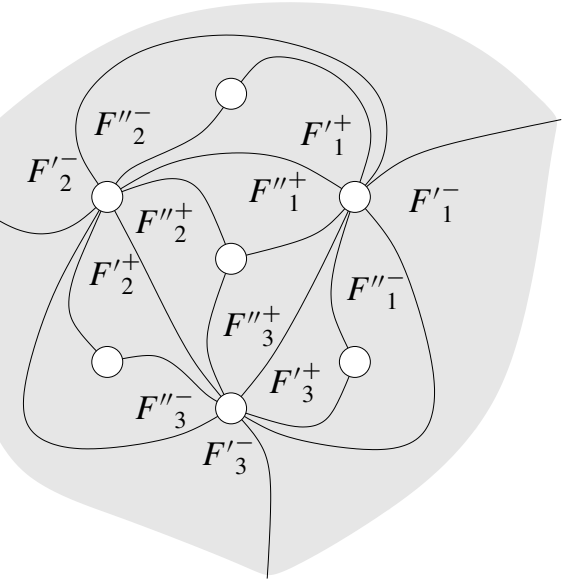

Figure 5. A polyhedral decomposition of $S^{3}-N(K)$. We are looking from inside of $B_{+}$. The white rectangles in (A) and (B) correspond to the attaching regions of the 2-handles. 


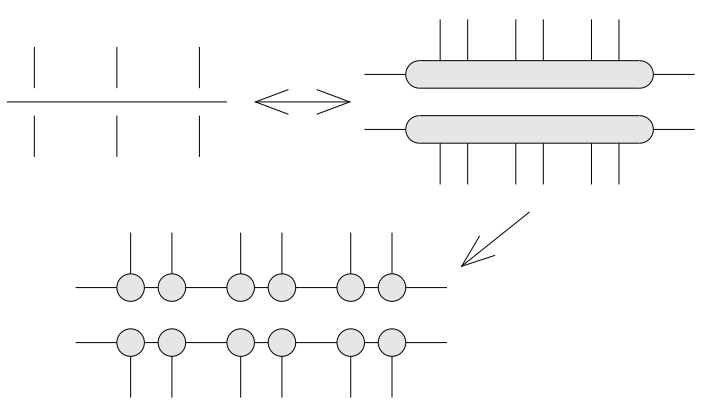

Figure 6. Stabilizations of the handle decomposition.

the Heegaard splitting; see Figure 6). We denote the new vertices, which originally belonged to $F_{i}^{ \pm}$, by ${F^{\prime}}_{i}^{ \pm}, F_{i}^{\prime \prime}, \ldots$ The dual of the graph gives a triangulation of $\partial B$ (Figures 5(D) and 5(E)). By abuse of notation, we denote the triangles dual to the vertices $F_{i}^{\prime \pm}, F^{\prime \prime}{ }_{i}^{ \pm}, \ldots$ by the same symbols. Taking a cone from an interior point of $B$, we obtain a triangulation $T$ of $B$ into $4 n$ tetrahedra. Regluing the triangles ${F^{\prime}}_{i}^{+}, F_{i}^{\prime \prime}, \ldots$ to ${F^{\prime}}_{i}^{-}, F_{i}^{\prime \prime}, \ldots$, we obtain a triangulation of $S^{3}-N(K)$ into $4 n$ tetrahedra, which was explained in [Weeks 2005]. We remark that this is not a triangulation in the usual sense: it is not a simplicial complex, and moreover, the link of some 0 -simplex is not homeomorphic to the 2-sphere. Actually there exist only three 0 -simplices; one is the cone point in $B$ (the north pole in [Weeks 2005]), the second is the 0 -simplex corresponding to the complementary regions of the diagram (the south pole), and the last one is a 0 -simplex whose small neighborhood is homeomorphic to the cone over the torus $\partial N(K)$.

Using this triangulation, we construct a triangulation of the cyclic branched covering $\widehat{C}_{l}$. Let $B_{0}, B_{1}, \ldots, B_{l-1}$ be $l$ copies of $B$ and let $T_{s}$ be the triangulation of $B_{s}$ we have constructed. We denote the triangles $F_{i}^{\prime \pm}, F_{i}^{\prime \prime}, \ldots$ on $\partial B_{s}$ by $F_{i, s}^{\prime \pm}, F_{i, s}^{\prime \prime}, \ldots$ respectively. By abuse of notation, we regard $F_{i, s}^{\prime \pm}, F_{i, s}^{\prime \prime}, \ldots$ simply as $F_{i, s}^{ \pm}$. Glue the $T_{s}$ 's along their boundary triangles by the identification maps

$$
F_{i, s}^{-} \rightarrow F_{i, s-1}^{+} \quad(i=1,2, \ldots, n, \quad s=0,1, \ldots, l-1) .
$$

Denote this triangulation by $\widehat{T}$. We define an ordering of each tetrahedron by assigning 0 to the interior vertex of $B_{s}, 1$ to the vertex corresponding to the complementary region of the diagram, 2 to the vertex corresponding to the under-crossing arc, and 3 to the vertex corresponding to the over-crossing arc, respectively (Figure 7). The orderings are compatible under the gluing maps. So $\widehat{T}$ is an ordered 3 -cycle. Here $\widehat{T}$ is a triangulation of $\widehat{C}_{l}$ except near the 0 -simplex corresponding to $K$, whose small neighborhood is homeomorphic to a cone over a torus. We can resolve this singularity by inserting a suspension of a $2 l$-gon around $K$ (Figure 8 ). As a 

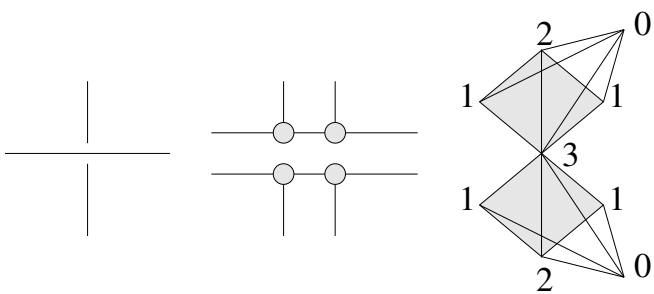

Figure 7. The orderings of the tetrahedra of $B$.

result, we obtain an ordered 3-cycle homeomorphic to $\widehat{C}_{l}$. (This procedure is called "blowing up" at a 0-simplex in [Neumann 2004].)

We construct a group cycle given by $\widehat{C}_{l}$ and a representation $\widehat{\rho}: \pi_{1}\left(\widehat{C}_{l}\right) \rightarrow G$ using the triangulation $\widehat{T}$. Here $\widehat{\rho}$ is given by the set $\left\{\widehat{\rho}\left(x_{i, s}\right)\right\} \subset G$ satisfying

$$
\begin{aligned}
& \widehat{\rho}\left(x_{k, s}\right)=\widehat{\rho}\left(x_{j, s-1}\right)^{-1} \widehat{\rho}\left(x_{i, s-1}\right) \widehat{\rho}\left(x_{j, s}\right), \\
& \widehat{\rho}\left(x_{1,0}\right)=\widehat{\rho}\left(x_{1,1}\right)=\widehat{\rho}\left(x_{1,2}\right)=\cdots=\widehat{\rho}\left(x_{1, l-1}\right)=1,
\end{aligned}
$$

with $i, j, k$ as in Figure 2 and $s=0,1, \ldots, l-1$. Give a labeling of vertices on $T_{s}$ for each $s$. Let $\left(g_{1}, g_{2}, g_{3}\right)$ be the labeling of vertices on $F_{i, s+1}^{-}$and let $\left(g_{1}^{\prime}, g_{2}^{\prime}, g_{3}^{\prime}\right)$ be the labeling of vertices on $F_{i, s}^{+}$. If these are related by

$$
\widehat{\rho}\left(x_{i, s}\right)\left(g_{1}, g_{2}, g_{3}\right)=\left(g_{1}^{\prime}, g_{2}^{\prime}, g_{3}^{\prime}\right),
$$

then we obtain a $G$-valued 1-cocycle $\lambda$ on $\widehat{T}$ by gluing $T_{s}$ using $\widehat{\rho}$. To obtain a group cycle given by $\widehat{C}_{l}$ and $\widehat{\rho}$, we insert a suspension over a $2 l$-gon to $\widehat{T}$ at the 0 -simplex corresponding to $K$. We give an ordering on the vertices of each tetrahedron of the suspension compatible with the ordering on the $2 l$-gon; for example, order the central 0-simplex maximal. These tetrahedra inherit a vertex labeling by $G$ on the boundary faces of the suspension. We assign any labeling at the central 0-simplex of the suspension. Then upper and lower tetrahedra have the same labelings with different orientations. So these cancel out in pairs and give no contribution to the group cycle represented by $\widehat{C}_{l}$ and $\widehat{\rho}$. Therefore the homology class given by $\widehat{T}$ and $\lambda$ represents the homology class given by $\widehat{C}_{l}$ and $\widehat{\rho}$.
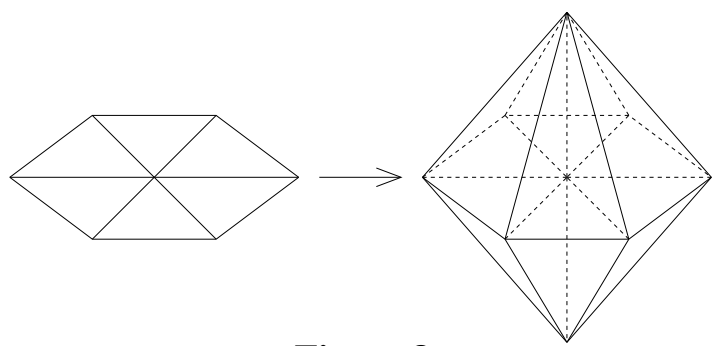

Figure 8 


\section{Quandle homology}

In this section, we review the definitions of quandles, rack (co)homology and quandle (co)homology. Our treatment of quandle (or rack) homology follows that of [Etingof and Graña 2003]. In Sections 5B and 5C, we recall the notions of colorings and quandle cocycle invariants defined in [Carter et al. 2003].

5A. Quandle and quandle homology. A quandle $X$ is a set with a binary operation * satisfying the following axioms:

(Q1) $x * x=x$ for any $x \in X$,

(Q2) the map $* y: X \rightarrow X$ defined by $x \mapsto x * y$ is a bijection for any $y \in X$, and

(Q3) $(x * y) * z=(x * z) *(y * z)$ for any $x, y, z \in X$.

We denote the inverse of $* y$ by $*^{-1} y$. For a quandle $X$, we define the associated group $G_{X}$ by

$$
G_{X}=\left\langle x \in X \mid y^{-1} x y=x * y(x, y \in X)\right\rangle .
$$

A quandle $X$ has a right $G_{X}$-action in the following way. Let $g=x_{1}^{\epsilon_{1}} x_{2}^{\epsilon_{2}} \cdots x_{n}^{\epsilon_{n}}$ be an element of $G_{X}$ where $x_{i} \in X$ and $\epsilon_{i}= \pm 1$. Define

$$
x * g=\left(\ldots\left(\left(x *^{\epsilon_{1}} x_{1}\right) *^{\epsilon_{2}} x_{2}\right) \ldots\right) *^{\epsilon_{n}} x_{n} .
$$

One can easily check that this is a right action of $G_{X}$ on $X$. So the free abelian group $\mathbb{Z}[X]$ generated by $X$ is a right $\mathbb{Z}\left[G_{X}\right]$-module.

Let $C_{n}^{R}(X)$ be the free left $\mathbb{Z}\left[G_{X}\right]$-module generated by $X^{n}$. We define the boundary map $C_{n}^{R}(X) \rightarrow C_{n-1}^{R}(X)$ by

$$
\begin{aligned}
& \partial\left(x_{1}, x_{2}, \ldots, x_{n}\right) \\
& \quad=\sum_{i=1}^{n}(-1)^{i}\left(\left(x_{1}, \ldots, \widehat{x_{i}}, \ldots, x_{n}\right)-x_{i}\left(x_{1} * x_{i}, \ldots, x_{i-1} * x_{i}, x_{i+1}, \ldots, x_{n}\right)\right) .
\end{aligned}
$$

Figure 9 shows a graphical picture of the boundary map. Let $C_{n}^{D}(X)$ be the $\mathbb{Z}\left[G_{X}\right]$-submodule of $C_{n}^{R}(X)$ generated by $\left(x_{1}, \ldots, x_{n}\right)$ with $x_{i}=x_{i+1}$ for some $i$. Now $C_{n}^{D}(X)$ is a subcomplex of $C_{n}^{R}(X)$. Let $C_{n}^{Q}(X)=C_{n}^{R}(X) / C_{n}^{D}(X)$. For a right $\mathbb{Z}\left[G_{X}\right]$-module $M$, we define the rack homology of $M$ by the homology of $C_{n}^{R}(X ; M)=M \otimes_{\mathbb{Z}\left[G_{X}\right]} C_{n}^{R}(X)$ and denote it by $H_{n}^{R}(X ; M)$. We also define the quandle homology of $M$ by the homology of $C_{n}^{Q}(X ; M)=M \otimes_{\mathbb{Z}\left[G_{X}\right]} C_{n}^{Q}(X)$ and denote it by $H_{n}^{Q}(X ; M)$. The homology $H_{n}^{Q}(X ; \mathbb{Z})$, where $\mathbb{Z}$ is the trivial $\mathbb{Z}\left[G_{X}\right]$ module, is equal to the usual quandle homology $H_{n}^{Q}(X)$. Let $Y$ be a set with a right $G_{X}$-action. For any abelian group $A$, the abelian group $A[Y]$ freely generated by $Y$ over $A$ is a right $\mathbb{Z}\left[G_{X}\right]$-module. The homology group $H_{n}^{Q}(X ; A[Y])$ is usually denoted by $H_{n}^{Q}(X ; A)_{Y}$ [Kamada 2007]. 


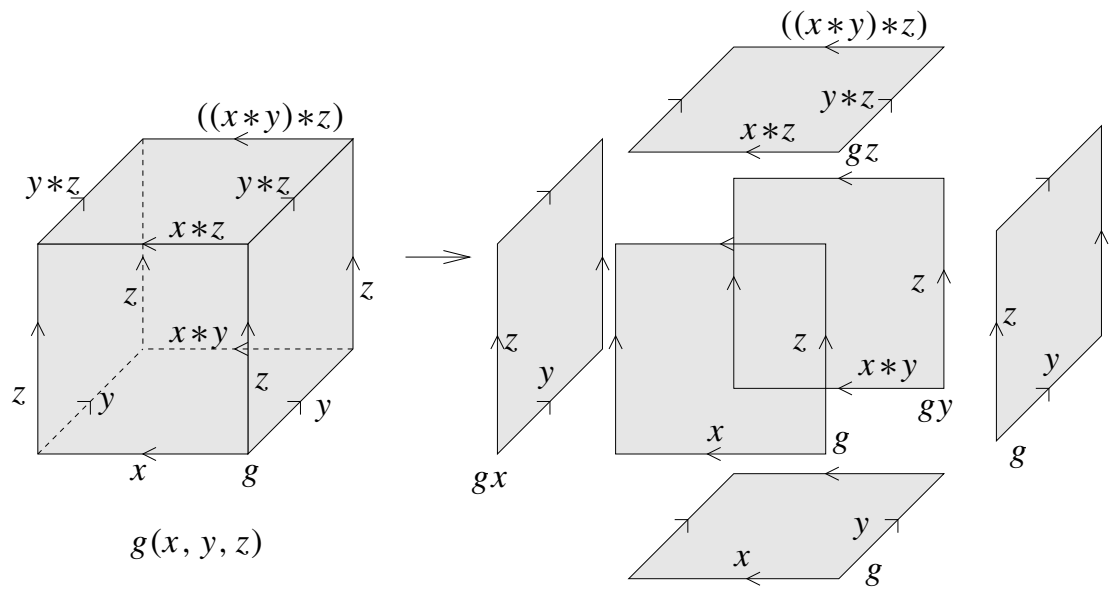

Figure 9. The boundary map $\partial(g(x, y, z))=-(g(y, z)-g x(y, z))+$ $(g(x, z)-g y(x * y, z))-(g(x, y)-g z(x * z, y * z))$. Here $x, y, z \in X$ and $g \in G_{X}$. Edges are labeled by elements of $X$ and vertices are labeled by elements of $G_{X}$.

Let $N$ be a left $\mathbb{Z}\left[G_{X}\right]$-module. We define the rack cohomology $H_{R}^{n}(X ; N)$ by the cohomology of $C_{R}^{n}(X ; N)=\operatorname{Hom}_{\mathbb{Z}\left[G_{X}\right]}\left(C_{n}^{R}(X), N\right)$. The quandle cohomology $H_{Q}^{n}(X ; N)$ is defined in a similar way. For a set $Y$ with a right $G_{X}$-action and an abelian group $A$, we let $\operatorname{Func}(Y, A)$ be the left $\mathbb{Z}\left[G_{X}\right]$-module generated by functions $\phi: Y \rightarrow A$, where the action is defined by $(g \phi)(y)=\phi(y g)$ for $y \in Y$ and $g \in G_{X}$. The cohomology group $H_{Q}^{n}(X ; \operatorname{Func}(Y, A))$ is usually denoted by $H_{Q}^{n}(X ; A)_{Y}$ [Kamada 2007].

5B. Shadow coloring and associated quandle cycle. Let $X$ be a quandle. Let $L$ be an oriented link in $S^{3}$ and let $D$ be a diagram of $L$. An arc coloring of $D$ is an assignment of elements of $X$ to $\operatorname{arcs}$ of $D$ satisfying the following relation at each crossing:

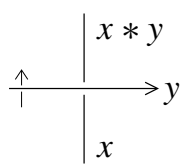

where $x, y \in X$. By the Wirtinger presentation of the knot complement, an arc coloring determines a representation of $\pi_{1}\left(S^{3}-L\right)$ into the associated group $G_{X}$. This is obtained by sending each meridian to its color.

Let $Y$ be a set with a right $G_{X}$ action. A region coloring of $D$ is an assignment of elements of $Y$ to regions of $D$ satisfying the relation

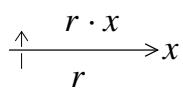


for any pair of adjacent regions, where $r \in Y$ and $x \in X$. A pair $\mathscr{Y}=(\mathscr{A}, \mathscr{R})$ is called a shadow coloring. If we fix a color of a region of $D$, then colors of other regions are uniquely determined. Therefore there always exists a region coloring compatible with a given arc coloring.

Define a cycle $C(\mathscr{Y})$ of $C_{2}^{Q}(X ; \mathbb{Z}[Y])$ for a shadow coloring $\mathscr{S}$. Put $+r \otimes(x, y)$ for a positive crossing and $-r \otimes(x, y)$ for a negative crossing colored by
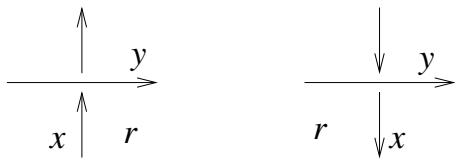

respectively. Then define

$$
C(\mathscr{S})=\sum_{c: \text { crossing }} \epsilon_{c} r_{c} \otimes\left(x_{c}, y_{c}\right) \in C_{2}^{Q}(X ; \mathbb{Z}[Y]),
$$

where $\epsilon_{c}= \pm 1$. We can easily check the following:

Proposition 5.1 [Inoue and Kabaya 2010]. $C(\mathscr{Y})$ is a cycle and the homology class $[C(\mathscr{S})]$ is invariant under Reidemeister moves. Moreover it does not depend on the choice of the region coloring if the action of $G_{X}$ on $Y$ is transitive.

So the homology class $[C(\mathscr{Y})]$ is an invariant of the arc coloring $\mathscr{A}$ in many cases. There are two important sets with right $G_{X}$-action: one is when $Y$ consists of one point $\{*\}$ and the other is when $Y=X$. Eisermann [2003; 2007] showed that the cycle $[C(\mathscr{Y})]$ for $Y=\{*\}$ is essentially described by the monodromy of some representation of the knot group along the longitude. So we concentrate on the invariant $[C(\mathscr{Y})]$ in the case of $Y=X$ from now on.

5C. Quandle cocycle invariant. Let $X$ be a quandle with $|X|<\infty$. Let $A$ be an abelian group and $f$ be a cocycle of $H_{Q}^{2}(X ; \operatorname{Func}(X, A))$. We define the (shadow) quandle cocycle invariant by

$$
\frac{1}{|X|} \sum_{\mathscr{S} \text { : shadow colorings }}\langle f, C(\mathscr{Y})\rangle \in \mathbb{Z}[A] .
$$

Here the sum is finite because there are only a finite number of shadow colorings of $D$. This is an invariant of oriented knots by Proposition 5.1. If $G_{X}$ acts on $X$ transitively (that is, $X$ is connected), $\langle f, C(\mathscr{S})\rangle$ does not depend on the choice of a region coloring $\mathscr{R}$ by Proposition 5.1, and thus

$$
\sum_{\substack{\mathscr{S}=(\mathscr{A}, \mathscr{R}), \mathscr{A}: \text { arc coloring }}}\langle f, C(\mathscr{S})\rangle
$$

coincides with the quandle cocycle invariant, where $\mathscr{R}$ is a region coloring compatible with $\mathscr{A}$. 
We can regard $f \in C_{Q}^{n}(X ; A)$ as an element of $C_{Q}^{n-1}(X ; \operatorname{Func}(X, A))$ by

$$
f\left(x_{1}, x_{2}, \ldots, x_{n-1}\right)(r)=f\left(r, x_{1}, x_{2}, \ldots, x_{n-1}\right) .
$$

This gives a homomorphism $H_{Q}^{n}(X ; A) \rightarrow H_{Q}^{n-1}(X ; \operatorname{Func}(X, A))$. Therefore a quandle 3-cocycle $f \in H_{Q}^{3}(X ; A)$ gives rise to a quandle cocycle invariant. Explicitly, the cocycle invariant has the form

$$
\sum_{\substack{\mathscr{S}=(\mathscr{A}, \mathscr{R}), \mathrm{s} \\ \mathcal{A}: \text { arc coloring }}} \sum_{c: \text { crossing }} \epsilon_{c} f\left(r_{c}, x_{c}, y_{c}\right) \in \mathbb{Z}[A],
$$

where $x_{c}, y_{c} \in X$ are given by $\mathscr{A}$ and $r_{c} \in X$ are given by $\mathscr{R}$.

\section{6. $H_{n}^{\Delta}(X ; \mathbb{Z})$ and the $\operatorname{map} H_{n}^{R}(X ; \mathbb{Z}[X]) \rightarrow H_{n+1}^{\Delta}(X ; \mathbb{Z})$}

Let $X$ be a quandle. Let $C_{n}^{\Delta}(X)=\operatorname{span}_{\mathbb{Z}}\left\{\left(x_{0}, \ldots, x_{n}\right) \mid x_{i} \in X\right\}$. We define the boundary operator of $C_{n}^{\Delta}(X)$ by

$$
\partial\left(x_{0}, \ldots, x_{n}\right)=\sum_{i=0}^{n}(-1)^{i}\left(x_{0}, \ldots, \widehat{x}_{i}, \ldots, x_{n}\right) .
$$

Since $X$ has a right action of $G_{X}$, the chain complex $C_{n}^{\Delta}(X)$ has a right action of $G_{X}$ by $\left(x_{0}, \ldots, x_{n}\right) * g=\left(x_{0} * g, \ldots, x_{n} * g\right)$. Let $M$ be a left $\mathbb{Z}\left[G_{X}\right]$-module. We denote the homology of $C_{n}^{\Delta}(X) \otimes_{\mathbb{Z}\left[G_{X}\right]} M$ by $H_{n}^{\Delta}(X ; M)$. For any abelian group $A$, we can also define the cohomology group $H_{\Delta}^{n}(X ; A)$ in a similar way.

Let $I_{n}$ be the set consisting of maps $\iota:\{1,2, \ldots, n\} \rightarrow\{0,1\}$. We let $|\iota|$ denote the cardinality of the set $\{i \mid \iota(i)=1,1 \leq i \leq n\}$. For each generator $r \otimes\left(x_{1}, x_{2}, \ldots, x_{n}\right)$ of $C_{n}^{R}(X ; \mathbb{Z}[X])$, where $r, x_{1}, \ldots, x_{n} \in X$, we define

$$
\begin{aligned}
r(\iota) & =r *\left(x_{1}^{\iota(1)} x_{2}^{\iota(2)} \cdots x_{n}^{\iota(n)}\right) \in X, \\
x(\iota, i) & =x_{i} *\left(x_{i+1}^{\iota(i+1)} x_{i+2}^{\iota(i+2)} \cdots x_{n}^{\iota(n)}\right) \in X,
\end{aligned}
$$

for any $\iota \in I_{n}$. Fix an element $q \in X$. For each $n \geq 1$, we define a homomorphism

$$
\varphi: C_{n}^{R}(X ; \mathbb{Z}[X]) \rightarrow C_{n+1}^{\Delta}(X) \otimes_{\mathbb{Z}\left[G_{X}\right]} \mathbb{Z}
$$

by

(6-1) $\varphi\left(r \otimes\left(x_{1}, x_{2}, \ldots, x_{n}\right)\right)=\sum_{\iota \in I_{n}}(-1)^{|\iota|}(q, r(\iota), x(\iota, 1), x(\iota, 2), \ldots, x(\iota, n))$.

For example, in the case $n=2$ (Figure 10),

$\varphi(r \otimes(x, y))$

$$
=(q, r, x, y)-(q, r * x, x, y)-(q, r * y, x * y, y)+(q,(r * x) * y, x * y, y),
$$



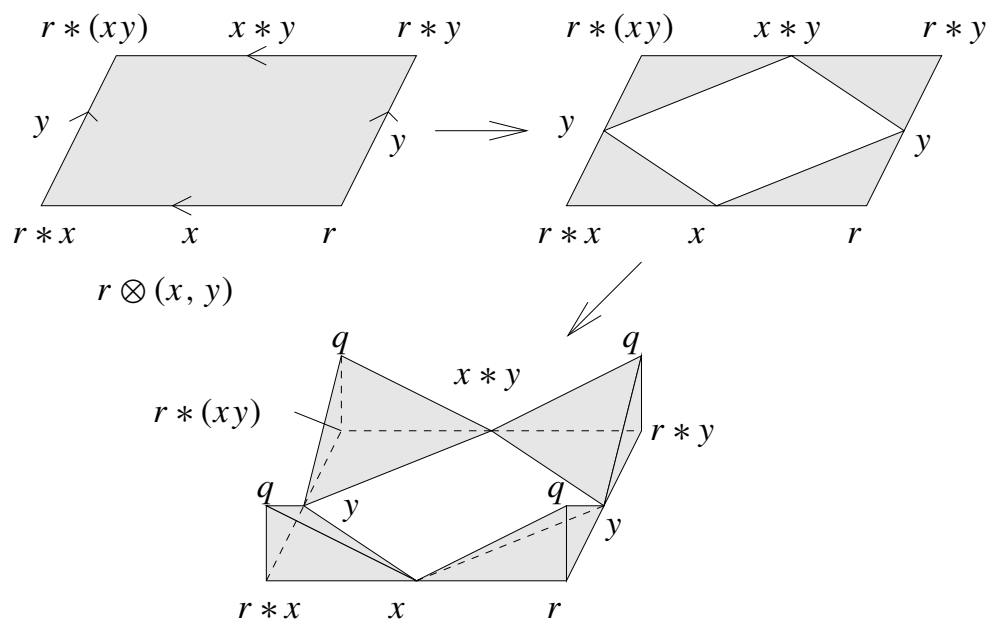

$(q, r, x, y)-(q, r * x, x, y)-(q, r * y, x * y, y)+(q, r *(x y), x * y, y)$

Figure 10

and in the case $n=3$,

$$
\begin{aligned}
\varphi(r \otimes(x, y, z))= & (q, r, x, y, z)-(q, r * x, x, y, z) \\
& -(q, r * y, x * y, y, z)-(q, r * z, x * z, y * z, z) \\
& +(q,(r * x) * y, x * y, y, z)+(q,(r * x) * z, x * z, y * z, z) \\
& +(q,(r * y) * z,(x * y) * z, y * z, z) \\
& -(q,((r * x) * y) * z,(x * y) * z, y * z, z) .
\end{aligned}
$$

Theorem 6.1 [Inoue and Kabaya 2010]. The map

$$
\varphi: C_{n}^{R}(X ; \mathbb{Z}[X]) \rightarrow C_{n+1}^{\Delta}(X) \otimes_{\mathbb{Z}\left[G_{X}\right]} \mathbb{Z}
$$

is a chain map.

Therefore $\varphi$ induces a homomorphism $\varphi_{*}: H_{n}^{R}(X ; \mathbb{Z}[X]) \rightarrow H_{n+1}^{\Delta}(X ; \mathbb{Z})$. We remark that the induced map $\varphi_{*}: H_{n}^{R}(X ; \mathbb{Z}[X]) \rightarrow H_{n+1}^{\Delta}(X ; \mathbb{Z})$ does not depend on the choice of $q \in X$ [Inoue and Kabaya 2010].

In general, it is easier to construct cocycles of $H_{n+1}^{\Delta}(X)$ from group cocycles of some group related to $X$ than those of $H_{n}^{R}(X ; \mathbb{Z}[X])$. If we have a function $f$ from $X^{k+1}$ to some abelian group $A$ satisfying
(1) $\sum_{i=0}^{k+1}(-1)^{i} f\left(x_{0}, \ldots, \widehat{x_{i}}, \ldots, x_{k+1}\right)=0$,
(2) $f\left(x_{0} * y, \ldots, x_{k} * y\right)=f\left(x_{0}, \ldots, x_{k}\right)$ for any $y \in X$, and
(3) $f\left(x_{0}, \ldots, x_{k}\right)=0$ if $x_{i}=x_{i+1}$ for some $i$, 
then $f$ is a cocycle of $H_{\Delta}^{k}(X ; A)$ and $\varphi^{*} f$ is a cocycle of $H_{Q}^{k-1}(X ; \operatorname{Func}(X, A))$. Moreover, $\varphi^{*} f$ can be regarded as a cocycle in $H_{Q}^{k}(X ; A)$ by

$$
\left(\varphi^{*} f\right)\left(r, x_{1}, \ldots, x_{k-1}\right)=\left(\varphi^{*} f\right)\left(x_{1}, \ldots, x_{k-1}\right)(r) .
$$

We will construct functions satisfying these three conditions from group cocycles.

\section{Cocycles of dihedral quandles}

For any integer $p>2$, let $R_{p}$ denote the cyclic group $\mathbb{Z} / p$ with quandle operation defined by $x * y=2 y-x \bmod p$. Actually this operation satisfies the quandle axioms. The quandle $R_{p}$ is called the dihedral quandle. In this section, we construct quandle cocycles of $R_{p}$ from group cocycles of $G=\mathbb{Z} / p$. In the next section, we will propose a general construction of quandle cocycles from group cocycles.

7A. Group cohomology of cyclic groups. Let $G$ be the cyclic group $\mathbb{Z} / p$ (where $p$ is an integer greater than 2). The first cohomology $H^{1}(G ; \mathbb{Z} / p)=\operatorname{Hom}(\mathbb{Z} / p, \mathbb{Z} / p)$ is generated by the 1 -cocycle $b_{1}$ defined by

$$
b_{1}(x)=x .
$$

The connecting homomorphism $\delta: H^{1}(G, \mathbb{Z} / p) \rightarrow H^{2}(G ; \mathbb{Z})$ of the long exact sequence corresponding to $0 \rightarrow \mathbb{Z} \rightarrow \mathbb{Z} \rightarrow \mathbb{Z} / p \rightarrow 0$ maps $b_{1}$ to a generator of $H^{2}(G ; \mathbb{Z})$, and the reduction $H^{2}(G ; \mathbb{Z}) \rightarrow H^{2}(G ; \mathbb{Z} / p)$ maps it to a generator $b_{2}$ of $H^{2}(G ; \mathbb{Z} / p)$. Explicitly, we have

$$
b_{2}(x, y)=\frac{1}{p}(\bar{y}-\overline{x+y}+\bar{x})= \begin{cases}1 & \text { if } \bar{x}+\bar{y} \geq p \\ 0 & \text { otherwise }\end{cases}
$$

where $\bar{x}$ is an integer $0 \leq \bar{x}<p$ such that $\bar{x} \equiv x \bmod p$. Cup products of $b_{1}$ 's and $b_{2}$ 's are also cocycles. Moreover, when $p$ is an odd prime, it is known that any element of $H^{*}(G ; \mathbb{Z} / p)$ can be presented by a cup product of $b_{1}$ 's and $b_{2}$ 's; see, for example, [Benson 1991, Proposition 3.5.5]. We remark that $b_{1}$ and $b_{2}$ and their products are normalized cocycles.

7B. Cocycle of $\boldsymbol{R}_{\boldsymbol{p}}$. For an integer $p>2$, let $f$ be a normalized $k$-cocycle of $H^{k}(G, \mathbb{Z} / p)$. Regarding $R_{p}$ as $G=\mathbb{Z} / p$, we obtain a map $f:\left(R_{p}\right)^{k+1} \rightarrow \mathbb{Z} / p$ satisfying
(1) $\sum_{i=0}^{k+1}(-1)^{i} f\left(x_{0}, \ldots, \widehat{x_{i}}, \ldots, x_{k+1}\right)=0$,
(3) $f\left(x_{0}, \ldots, x_{k}\right)=0$ if $x_{i}=x_{i+1}$ for some $i$,

by using homogeneous notation (Section 2A). If $f$ also satisfies the condition

(2) $f\left(x_{0} * y, \ldots, x_{k} * y\right)=f\left(x_{0}, \ldots, x_{k}\right)$ for any $y \in R_{p}$, 
then $f$ gives rise to a quandle $k$-cocycle of $H_{Q}^{k}\left(R_{p} ; \mathbb{Z} / p\right)$ by the construction of Section 6. Define $\tilde{f}:\left(R_{p}\right)^{k+1} \rightarrow \mathbb{Z} / p$ by

$$
\tilde{f}\left(x_{0}, \ldots, x_{k}\right)=f\left(x_{0}, \ldots, x_{k}\right)+f\left(-x_{0}, \ldots,-x_{k}\right) .
$$

Then $\tilde{f}$ satisfies condition (2) by the left invariance of the homogeneous group cocycle. It is easy to check that $\tilde{f}$ also satisfies conditions (1) and (3). So we obtain a quandle $k$-cocycle.

We give an explicit presentation of the 3-cocycle arising from $b_{1} b_{2} \in H^{3}(G ; \mathbb{Z} / p)$. Let

$$
d(x, y)=b_{2}(x, y)-b_{2}(-x,-y)
$$

then $d$ is a 2-cocycle. (We can check that $d$ is cohomologous to $2 b_{2}$.) Then by the defining equation $(7-2), \widetilde{b_{1} b_{2}}$ is given by

$$
[x|y| z] \mapsto x \cdot d(y, z) .
$$

By definition we have

$$
d(-x,-y)=-d(x, y)
$$

and

$$
d(x, y)=\left\{\begin{aligned}
1 & \text { if } \bar{x}+\bar{y}>p, \\
-1 & \text { if } \bar{x}+\bar{y}<p, x \neq 0 \text { and } y \neq 0 \\
0 & \text { otherwise. }
\end{aligned}\right.
$$

We remark that the cocycle $d$ can be easily understood geometrically. Identify $i \in$ $\mathbb{Z} / p$ with the complex number $\zeta^{i}$, where $\zeta=\exp (2 \pi \sqrt{-1} / p)$. Then $d(x, y)=-1$ if $(0, x, x+y)$ is counterclockwise, $d(x, y)=+1$ if $(0, x, x+y)$ is clockwise, and $d(x, y)=0$ if $(0, x, x+y)$ is degenerate (Figure 11). This interpretation and Equation (7-3) make various calculations easy.

Proposition 7.1. The quandle 3-cocycle arising from $b_{1} b_{2} \in H^{3}(G ; \mathbb{Z} / p)$ has the following presentation:

$$
(x, y, z) \mapsto 2 z(d(y-x, z-y)+d(y-x, y-z)), \quad\left(x, y, z \in R_{p}\right) .
$$

This is a nontrivial quandle 3-cocycle of $R_{p}$ with $\mathbb{Z} / p$ coefficients.
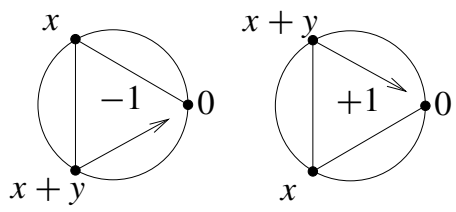

Figure 11. The value of $d(x, y)$. 
Proof. In (6-1), since the map $\varphi_{*}$ does not depend on the choice of $q \in R_{p}$, we let $q=0$. Then we have

$$
\begin{aligned}
\varphi(x, y, z)= & (0, x, y, z)-(0, x * y, y, z) \\
& -(0, x * z, y * z, z)+(0,(x * y) * z, y * z, z) \\
= & (0, x, y, z)-(0,2 y-x, y, z) \\
& -(0,2 z-x, 2 z-y, z)+(0,2 z-2 y+x, 2 z-y, z),
\end{aligned}
$$

for $x, y, z \in R_{p}$. Rewriting in inhomogeneous notation, this is equal to

$$
\begin{aligned}
& {[x|y-x| z-y]-[2 y-x|x-y| z-y]} \\
& \quad-[2 z-x|x-y| y-z]+[2 z-2 y+x|y-x| y-z] .
\end{aligned}
$$

The evaluation of $\widetilde{b_{1} b_{2}}$ on this chain is

$$
\begin{array}{rl} 
& x \cdot d(y-x, z-y)-(2 y-x) d(x-y, z-y) \\
& \quad-(2 z-x) d(x-y, y-z)+(2 z-2 y+x) d(y-x, y-z) \\
=x & d(y-x, z-y)+(2 y-x) d(y-x, y-z) \\
& \quad+(2 z-x) d(y-x, z-y)+(2 z-2 y+x) d(y-x, y-z) \\
= & 2 z \cdot d(y-x, z-y)+2 z \cdot d(y-x, y-z) .
\end{array}
$$

We will see that this cocycle is nontrivial because the evaluation on the cycle given by a shadow coloring $\mathscr{S}$ of the $(2, p)$-torus link (Figure 12) is nonzero. Color two arcs by $x, y \in R_{p}$ as in Figure 12. Then other arcs must be colored by $(i+1) y-i x$ by the relations at the crossings. Let $r$ be the color of the central region. Then we have

$$
C(\mathscr{S})=\sum_{i=0}^{p-1} r \otimes(i y-(i-1) x,(i+1) y-i x) .
$$

We assume that $r=0$ and $x=0$. Evaluation of the cocycle on $C(\mathscr{S})$ yields

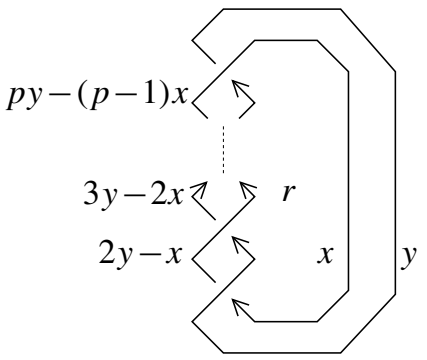

Figure 12. A shadow coloring of the $(2, p)$-torus knot by $R_{p}$ (for any $\left.x, y, r \in R_{p}\right)$. 


$$
\begin{aligned}
& \sum_{i=0}^{p-1} 2(i+1) y(d(i y, y)+d(i y,-y)) \\
& =2 y \sum_{i=0}^{p-1}(i+1)(d(i y, y)-d(-i y, y)) \quad(\text { by }(7-3)) \\
& =2 y \sum_{i=0}^{p-1}((i+1) d(i y, y)+(i-1) d(i y, y))=4 y \sum_{i=0}^{p-1} i \cdot d(i y, y) .
\end{aligned}
$$

By the next lemma, this is equal to $-4 y^{2} \bmod p$.

Lemma 7.2. Let $p>2$ be an integer. For $0<y<p$, we have

$$
\sum_{i=0}^{p-1} i \cdot d(i y, y)= \begin{cases}-y & p: \text { odd } \\ \frac{p}{2}-y & p: \text { even } .\end{cases}
$$

When $y=0$, the left-hand side is 0 .

Proof. When $y=0$, this is straightforward since $d(x, 0)=0$ for any $0 \leq x<p$. When $y$ is a unit in $\mathbb{Z} / p$, by (7-4) we have

$$
\begin{aligned}
\sum_{i=0}^{p-1} i \cdot d(i y, y) & =\sum_{i=0}^{p-1} \frac{i}{y} \cdot d(i, y) \\
& =\frac{1}{y}(-1-2-\cdots-(p-y-1)+(p-y+1)+(p-y+2)+\cdots+(p-1)) \\
& =\frac{1}{y}\left(\frac{(p-y-1)(y-p)}{2}+\frac{(y-1)(2 p-y)}{2}\right) \\
& =\frac{1}{y}\left(-y^{2}-\frac{p}{2}+2 p y-\frac{p^{2}}{2}\right) \equiv-y-\frac{p}{2} \cdot \frac{1}{y} .
\end{aligned}
$$

This is equal to $-y$ when $p$ is odd. When $p$ is even, $-p / 2 \cdot 1 / y \equiv p / 2 \bmod p$ since $1 / y$ is a unit in $\mathbb{Z} / p$.

When $y$ is not a unit in $\mathbb{Z} / p$, let $c$ be the greatest common divisor of $y$ and $p$. Then

$$
\sum_{i=0}^{p-1} i \cdot d(i y, y)=c \sum_{j=0}^{p / c-1} \frac{j}{y / c} \cdot d(j c, y)=\frac{c}{y / c} \sum_{j=0}^{p / c-1} j \cdot d(j, y / c) .
$$

Since $y / c$ is a unit in $\mathbb{Z} /(p / c)$, this reduces to the previous case.

Since 2 is divisible in $\mathbb{Z} / p$ when $p$ is odd, we have:

Corollary 7.3. When $p>2$ is an odd number,

$$
(x, y, z) \mapsto z(d(y-x, z-y)+d(y-x, y-z))
$$

is a nontrivial quandle 3-cocycle of $R_{p}$ with $\mathbb{Z} / p$ coefficients. 
When $p$ is prime, it is known that $\operatorname{dim}_{\mathbb{F}_{p}} H_{Q}^{3}\left(R_{p} ; \mathbb{F}_{p}\right)=1$. Therefore our cocycle is a constant multiple of the Mochizuki 3-cocycle [2003]. We remark that when $p$ is prime, $\operatorname{dim}_{\mathbb{F}_{p}} H_{Q}^{n}\left(R_{p} ; \mathbb{F}_{p}\right)$ was calculated for any $n$ by Nosaka [2009], who gave a system of generators of $H_{Q}^{n}\left(R_{p} ; \mathbb{F}_{p}\right)$.

When $p$ is an odd integer, Nosaka [2010] showed that $H_{3}^{Q}\left(R_{p} ; \mathbb{Z}\right) \cong \mathbb{Z} / p$. Since $H_{2}^{Q}\left(R_{p} ; \mathbb{Z}\right)$ is zero, we have $H_{Q}^{3}\left(R_{p} ; \mathbb{Z} / p\right) \cong \mathbb{Z} / p$. This means that there exists a nontrivial quandle 3 -cocycle of $R_{p}$ with $\mathbb{Z} / p$ coefficients.

\section{General construction}

In this section, we generalize the construction in the previous section to wider classes of quandles. We construct a quandle cocycle of a faithful homogeneous quandle $X$ from a group cocycle of $\operatorname{Aut}(X)$ when an obstruction living in the second cohomology of $\operatorname{Aut}(X)$ vanishes.

8A. Let $G$ be a group. Fix an element $h \in G$. Let $\operatorname{Conj}(h)=\left\{g^{-1} h g \mid g \in G\right\}$. Now $\operatorname{Conj}(h)$ has a quandle operation by $x * y=y^{-1} x y$. In this section, we construct a quandle cocycle of $\operatorname{Conj}(h)$ from a group cocycle of $G$. First we shall show that this class of quandles is not so special.

Let $X$ be a quandle. We denote the group of the quandle automorphisms of $X$ by $\operatorname{Aut}(X)$. We consider an automorphism that acts on $X$ from the right. For $x \in X$, let $S(x)$ be the map that sends $y$ to $y * x$. By the axioms (Q2) and (Q3), $S(x)$ is a quandle automorphism. $X$ is called faithful if $S: X \rightarrow \operatorname{Aut}(X)$ is injective. A quandle $X$ is homogeneous if $\operatorname{Aut}(X)$ acts on $X$ transitively. The following lemma was essentially shown in Theorem 7.1 of [Joyce 1982], but we include a proof for completeness.

Lemma 8.1. Every faithful homogeneous quandle $X$ is represented by $\operatorname{Conj}(h)$ with some group $G$ and $h \in G$.

Proof. For $x \in X$ and $g \in \operatorname{Aut}(X)$, we have $S(x g)=g^{-1} S(x) g$. In fact, $(y) S(x g)=$ $y *(x g)=\left(y g^{-1} * x\right) g=(y) g^{-1} S(x) g$ for any $y \in X$.

Let $G=\operatorname{Aut}(X)$ and fix an element $x_{0} \in X$. Put $h=S\left(x_{0}\right)$. Because $X$ is homogeneous, for any $x \in X$, there exists $g \in G$ such that $x=x_{0} g$. So we have $S(x)=S\left(x_{0} g\right)=g^{-1} h g$, that is, $S(x) \in \operatorname{Conj}(h)$. Therefore we obtain a homomorphism $S: X \rightarrow \operatorname{Conj}(h)$. This is surjective since $g^{-1} S\left(x_{0}\right) g=S\left(x_{0} g\right)$, and injective since $X$ is faithful.

Let $Z(h)=\{g \in G \mid g h=h g\}$ be the centralizer of $h$ in $G$.

Lemma 8.2. A map

$$
\begin{array}{ccc}
\operatorname{Conj}(h) & \rightarrow & Z(h) \backslash G \\
\Psi & & \Psi \\
g^{-1} h g & \mapsto & Z(h) g
\end{array}
$$


is well-defined and bijective.

Proof. Let $g_{1}^{-1} h g_{1}=g_{2}^{-1} h g_{2}$. Then $\left(g_{1} g_{2}^{-1}\right)^{-1} h\left(g_{1} g_{2}^{-1}\right)=h$, so $g_{1} g_{2}^{-1} \in Z(h)$ and $g_{1} \in Z(h) g_{2}$. This means that $g_{1}$ and $g_{2}$ belong to the same right coset. Therefore the map is well-defined. By a similar argument, we can show the injectivity. Surjectivity is trivial by definition.

Now we study the quandle structure on $Z(h) \backslash G$ and construct a section of the projection $\pi: G \rightarrow Z(h) \backslash G$. The quandle operation on $\operatorname{Conj}(h)$ induces a quandle operation on $Z(h) \backslash G$ :

$$
\begin{aligned}
Z(h) g_{1} * Z(h) g_{2} \leftrightarrow\left(g_{1}^{-1} h g_{1}\right) & *\left(g_{2}^{-1} h g_{2}\right)=\left(g_{2}^{-1} h g_{2}\right)^{-1}\left(g_{1}^{-1} h g_{1}\right)\left(g_{2}^{-1} h g_{2}\right) \\
& =\left(g_{1} g_{2}^{-1} h g_{2}\right)^{-1} h\left(g_{1} g_{2}^{-1} h g_{2}\right) \leftrightarrow Z(h) g_{1}\left(g_{2}^{-1} h g_{2}\right) .
\end{aligned}
$$

This quandle operation on $Z(h) \backslash G$ lifts to a quandle operation $\widetilde{*}$ on $G$ by

$$
g_{1} \tilde{*} g_{2}=h^{-1} g_{1}\left(g_{2}^{-1} h g_{2}\right), \quad\left(g_{1}, g_{2} \in G\right) .
$$

We can easily check that $\widetilde{*}$ satisfies the quandle axioms (the inverse operation is given by $\left.g_{1} \widetilde{*}^{-1} g_{2}=h g_{1} g_{2}^{-1} h^{-1} g_{2}\right)$ and that the projection map $\pi: G \rightarrow Z(h) \backslash G$ is a quandle homomorphism. We remark that the quandle operation given by (8-1) has been already studied by Joyce [1982] and Eisermann [2003].

Let $s: Z(h) \backslash G \rightarrow G$ be a section of $\pi$, that is, a map (not a homomorphism) satisfying $\pi \circ s=\mathrm{id}$. Since $s(x * y)$ and $s(x) \widetilde{*} s(y)$ are in the same coset in $Z(h) \backslash G$, there exists an element $c(x, y) \in Z(h)$ satisfying

$$
s(x) \widetilde{*} s(y)=c(x, y) s(x * y) .
$$

Lemma 8.3. If $Z(h)$ is an abelian group, $c: Z(h) \backslash G \times Z(h) \backslash G \rightarrow Z(h)$ is a quandle 2-cocycle. If the cocycle $c$ is cohomologous to zero, we can change the section s to satisfy $s(x * y)=s(x) \widetilde{*} s(y)$.

Proof. For $c_{1}, c_{2} \in Z(h)$ and $g_{1}, g_{2} \in G$, we have

$$
\left(c_{1} g_{1}\right) \widetilde{*}\left(c_{2} g_{2}\right)=h^{-1} c_{1} g_{1} g_{2}^{-1} c_{2}^{-1} h c_{2} g_{2}=c_{1} h^{-1} g_{1} g_{2}^{-1} h g_{2}=c_{1}\left(g_{1} \approx g_{2}\right) \text {. }
$$

Therefore,

$$
\begin{aligned}
(s(x) \tilde{*} s(y)) \widetilde{*} s(z) & =(c(x, y) s(x * y)) \widetilde{*} s(z) \\
& =c(x, y)(s(x * y) \widetilde{*} s(z)) \\
& =c(x, y) c(x * y, z) s((x * y) * z)
\end{aligned}
$$

and

$$
\begin{aligned}
(s(x) \widetilde{*}(z)) \widetilde{*}(s(y) \widetilde{*}(z)) & =(c(x, z) s(x * z)) \widetilde{*}(c(y, z) s(y * z)) \\
& =c(x, z) c(x * z, y * z) s((x * z) *(y * z))
\end{aligned}
$$


for any $x, y, z \in Z(h) \backslash G$. Comparing (8-2) and (8-3), we have

$$
c(x, z) c(x * y, z)^{-1} c(x, y)^{-1} c(x * z, y * z)=1 .
$$

By $s(x) \widetilde{*} s(x)=s(x)$, we also have $c(x, x)=1$.

If $c$ is cohomologous to zero; then there exists a map $b: Z(h) \backslash G \rightarrow Z(h)$ satisfying $c(x, y)=b(x) b(x * y)^{-1}$. Put $s^{\prime}(x)=b(x)^{-1} s(x)$, then $s^{\prime}$ satisfies $s^{\prime}(x) \widetilde{*} s^{\prime}(y)=s^{\prime}(x * y)$.

Remark 8.4. The 2-cocycle $c$ has already appeared in [Eisermann 2003] in a similar context.

Example 8.5. Let $G$ be the dihedral group

$$
D_{2 p}=\left\langle h, x \mid h^{2}=x^{p}=h x h x=1\right\rangle,
$$

where $p$ is an odd number greater than 2. Then we have $Z(h)=\{1, h\}$ and $\operatorname{Conj}(h)=\left\{x^{-i} h x^{i} \mid i=0,1, \ldots, p-1\right\}=\left\{h x^{2 i} \mid i=0, \ldots, p-1\right\}$. We can identify $x^{-i} h x^{i} \in \operatorname{Conj}(h)$ with $i \in R_{p}=\{0,1,2, \ldots, p-1\}$. Define a section $s: Z(h) \backslash G \rightarrow G$ by

$$
\begin{aligned}
& \operatorname{Conj}(h) \cong Z(h) \backslash G \stackrel{s}{\rightarrow} \quad G \\
& \Psi \quad \Psi \quad \cup \\
& x^{-i} h x^{i} \leftrightarrow Z(h) x^{i} \mapsto h x^{i}
\end{aligned}
$$

Then we have

$$
\begin{aligned}
s\left(Z(h) x^{i} * Z(h) x^{j}\right) & =s\left(Z(h) x^{2 j-i}\right)=h x^{2 j-i} \\
& =h^{-1}\left(h x^{i}\right)\left(x^{-j} h x^{j}\right)=s\left(Z(h) x^{i}\right) \widetilde{*} s\left(Z(h) x^{j}\right) .
\end{aligned}
$$

Therefore $c(x, y)=0$ for any $x, y \in R_{p}$.

8B. Let $G$ be a group. Fix $h \in G$ with $h^{l}=1(l>1)$. In the following we assume:

Assumption 8.6. $Z(h)$ is abelian and the 2-cocycle corresponding to $G \rightarrow Z(h) \backslash G$ is cohomologous to zero.

Under this assumption, we can take a section $s: Z(h) \backslash G \rightarrow G$ satisfying

$$
s(x * y)=s(x) \widetilde{*}(y),
$$

by Lemma 8.3. Let $f: G^{k+1} \rightarrow A$ be a normalized group $k$-cocycle of $G$ in the homogeneous notation, where $A$ is an abelian group. Then $f$ satisfies
(1) $\sum_{i=0}^{k+1}(-1)^{i} f\left(x_{0}, \ldots, \widehat{x_{i}}, \ldots, x_{k+1}\right)=0$,
(2) $f\left(g x_{0}, \ldots, g x_{k}\right)=f\left(x_{0}, \ldots, x_{k}\right)$ for any $g \in G$ (left invariance), and
(3) $f\left(x_{0}, \ldots, x_{k}\right)=0$ if $x_{i}=x_{i+1}$ for some $i$. 
Since it is convenient to use a right-invariant function in the following construction, we modify condition (2) by replacing $f\left(x_{0}, \ldots, x_{k}\right)$ with $f\left(x_{0}^{-1}, \ldots, x_{k}^{-1}\right)$ :

(2') $f\left(x_{0} g, \ldots, x_{k} g\right)=f\left(x_{0}, \ldots, x_{k}\right)$ for any $g \in G$ (right invariance).

Define $\tilde{f}: \operatorname{Conj}(h)^{k+1} \rightarrow A$ by

$$
\tilde{f}\left(x_{0}, \ldots, x_{k}\right)=\sum_{i=0}^{l-1} f\left(h^{i} s\left(x_{0}\right), \ldots, h^{i} s\left(x_{k}\right)\right),
$$

for $x_{0}, \ldots, x_{k} \in \operatorname{Conj}(h)$.

Proposition 8.7. The function $\tilde{f}$ satisfies conditions (1), (2) and (3) of Section 6. Therefore $\tilde{f}$ gives rise to a $k$-cocycle of $H_{\Delta}^{k}(\operatorname{Conj}(h) ; A)$.

Proof. It is clear that (1) and (3) are satisfied from the conditions on a normalized group cocycle in homogeneous notation. We only have to check the second property. $\tilde{f}\left(x_{0} * y, \ldots, x_{k} * y\right)$

$$
\begin{aligned}
& =\sum_{i=0}^{l-1} f\left(h^{i} s\left(x_{0} * y\right), \ldots, h^{i} s\left(x_{k} * y\right)\right) \\
& =\sum_{i=0}^{l-1} f\left(h^{i} s\left(x_{0}\right) \widetilde{*} s(y), \ldots, h^{i} s\left(x_{k}\right) \widetilde{*} s(y)\right) \\
& =\sum_{i=0}^{l-1} f\left(h^{i-1} s\left(x_{0}\right)\left(s(y)^{-1} h s(y)\right), \ldots, h^{i-1} s\left(x_{k}\right)\left(s(y)^{-1} h s(y)\right)\right) \\
& =\sum_{i=0}^{l-1} f\left(h^{i-1} s\left(x_{0}\right), \ldots, h^{i-1} s\left(x_{k}\right)\right) \quad(\text { right invariance }) \\
& =\tilde{f}\left(x_{0}, \ldots, x_{k}\right) .
\end{aligned}
$$

Combining with the arguments of Section 6, we have:

Corollary 8.8. If $Z(h)$ is abelian and the 2-cocycle corresponding to $G \rightarrow Z(h) \backslash G$ is cohomologous to zero, then there is a homomorphism

$$
H^{n}(G ; A) \rightarrow H_{Q}^{n}(\operatorname{Conj}(h) ; A)
$$

for any abelian group A.

8C. We return to the case of $R_{p}$ discussed in the previous section. We assume that $p$ is an odd integer greater than 2 . Let $G$ be the dihedral group

$$
D_{2 p}=\left\langle h, x \mid h^{2}=x^{p}=h x h x=1\right\rangle .
$$


Consider the short exact sequence

$$
0 \rightarrow \mathbb{Z} / p \rightarrow D_{2 p} \rightarrow \mathbb{Z} / 2 \rightarrow 0 .
$$

We regard $\mathbb{Z} / 2$ as $\{1, h\}$ by taking coset representatives in $D_{2 p}$. Then $\mathbb{Z} / 2$ acts on $\mathbb{Z} / p$ by $h\left(x^{i}\right)=x^{-i}$. This induces the restriction map

$$
H^{*}\left(D_{2 p} ; \mathbb{Z} / p\right) \rightarrow H^{*}(\mathbb{Z} / p ; \mathbb{Z} / p)^{\mathbb{Z} / 2} .
$$

We can show that this homomorphism is an isomorphism [Brown 1982, Proposition III.10.4]. To obtain a group cocycle of $D_{2 p}$ from a group cocycle of $\mathbb{Z} / p$, we need the inverse map, which is called the transfer. The transfer map is described as follows (see also [Brown 1982]). Let $r$ be the map $D_{2 p} \rightarrow \mathbb{Z} / p$ defined by $r\left(x^{i}\right)=x^{i}$ and $r\left(h x^{i}\right)=x^{-i}$. For a cocycle $f$ of $H^{n}(\mathbb{Z} / p ; \mathbb{Z} / p)^{\mathbb{Z} / 2}$, the image $f^{\prime}$ of the transfer is given by

$$
f^{\prime}\left(x_{0}, \ldots, x_{n}\right)=f\left(r x_{0}, \ldots, r x_{n}\right)+f\left(h r x_{0}, \ldots, h r x_{n}\right)
$$

in homogeneous notation. When restricted to the image of $s: Z(h) \backslash G \rightarrow G$, this is equal to the map defined in (7-2). Applying our construction for this group cocycle, we obtain a quandle 3 -cocycle of $R_{p}$, which is twice the cocycle constructed in the previous section.

8D. We end this section by giving another homomorphism from $H^{n}(G ; A)$ to $H_{Q}^{n}(\operatorname{Conj}(h) ; A)$ arising from a more general context.

Let $X$ be a quandle and let $M$ be a right $\mathbb{Z}\left[G_{X}\right]$-module. We can construct a map from the rack homology $H_{n}^{R}(X ; M)$ to the group homology $H_{n}\left(G_{X} ; M\right)$. The following lemma is well-known; for example, see Lemma 7.4 of Chapter I of [Brown 1982].

Lemma 8.9. Let $\cdots \rightarrow P_{1} \rightarrow P_{0} \rightarrow M \rightarrow 0$ be a chain complex where the $P_{i}$ are projective (for example, free). Let $\cdots \rightarrow C_{1} \rightarrow C_{0} \rightarrow N \rightarrow 0$ be an acyclic complex. Any homomorphism $M \rightarrow N$ can be extended to a chain map from $\left\{P_{*}\right\}$ to $\left\{C_{*}\right\}$. Moreover, such a chain map is unique up to chain homotopy.

So there exists a unique chain map from $C_{*}^{R}(X)$ to $C_{*}\left(G_{X}\right)$ up to homotopy. This map induces $M \otimes_{\mathbb{Z}\left[G_{X}\right]} C_{*}^{R}(X) \rightarrow M \otimes_{\mathbb{Z}\left[G_{X}\right]} C_{*}\left(G_{X}\right)$ and then $H_{n}^{R}(X ; M) \rightarrow$ $H_{n}\left(G_{X} ; M\right)$. Using normalized chains in group homology, we can also construct a map $H_{n}^{Q}(X ; M) \rightarrow H_{n}\left(G_{X} ; M\right)$. We give an explicit chain map. Let $\left(x_{1}, \ldots, x_{n}\right)$ be a generator of $C_{n}^{R}(X)$. We define a map $\psi$ by

$$
\psi\left(\left(x_{1}, \ldots, x_{n}\right)\right)=\sum_{\sigma \in \mathfrak{S}_{n}} \operatorname{sgn}(\sigma)\left[y_{\sigma, 1}|\cdots| y_{\sigma, i}|\cdots| y_{\sigma, n}\right],
$$

where $y_{\sigma, i} \in X$ is defined for a permutation $\sigma$ and $i \in\{1, \ldots, n\}$ as follows. Let 


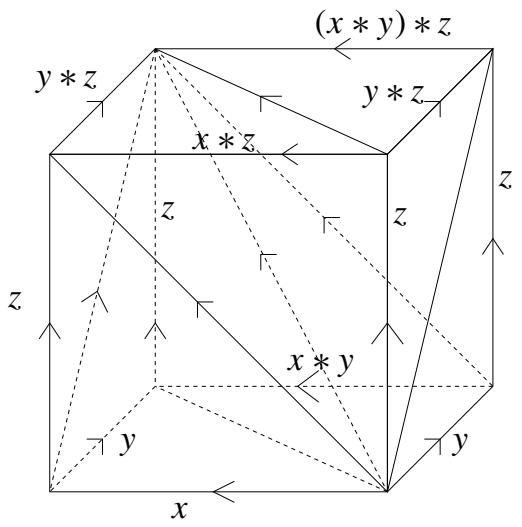

Figure 13

$j_{1}, \ldots, j_{i}<i$ be the maximal set of numbers satisfying

$$
\sigma(i)<\sigma\left(j_{1}\right)<\sigma\left(j_{2}\right)<\cdots<\sigma\left(j_{i}\right)
$$

Then define

$$
y_{\sigma, i}=x_{\sigma(i)} *\left(x_{\sigma\left(j_{1}\right)} x_{\sigma\left(j_{2}\right)} \cdots x_{\sigma\left(j_{i}\right)}\right) .
$$

A graphical picture of this map is given in Figure 13. For example, when $n=3$,

$$
\begin{aligned}
\psi((x, y, z)) & =[x|y| z]-[x|z| y * z] \\
+ & {[y|z|(x * y) * z]-[y|x * y| z]+[z|x * z| y * z]-[z|y * z|(x * y) * z], }
\end{aligned}
$$

for $(x, y, z) \in C_{3}^{R}(X)$. Dually, we also have a map $H^{n}\left(G_{X} ; M\right) \rightarrow H_{Q}^{n}(X ; M)$.

We apply this map for $\operatorname{Conj}(h)$. Since there exists a natural homomorphism from the associated group $G_{\operatorname{Conj}(h)}$ to $G$, we have a homomorphism

$$
H^{n}(G ; A) \rightarrow H^{n}\left(G_{\operatorname{Conj}(h)} ; A\right) \rightarrow H_{Q}^{n}(\operatorname{Conj}(h) ; A) .
$$

Fenn, Rourke and Sanderson [Fenn et al. 1995] defined the rack space $B X$. Since $\pi_{1}(B X)$ is isomorphic to $G_{X}$, there exists a unique map, up to homotopy, from $B X$ to the Eilenberg-MacLane space $K\left(G_{X}, 1\right)$ that induces the isomorphism between their fundamental groups. This map induces a homomorphism $H^{n}\left(G_{X} ; M\right) \rightarrow H_{Q}^{n}(X ; M)$, which is equal to the map we have constructed in this subsection. Clauwens [2011, Proposition 25] showed that this map vanishes under some conditions on $X$ and $M$. In particular, when $p$ is odd prime,

$$
H^{n}\left(D_{2 p} ; \mathbb{Z} / p\right) \rightarrow H_{Q}^{n}\left(R_{p} ; \mathbb{Z} / p\right)
$$

vanishes for $n>0$. 


\section{Quandle cycle and branched cover}

In this section, we study the dual of the previous construction. We show that the cycle $C(\mathscr{Y})$ associated with a shadow coloring $\mathscr{Y}=(\mathscr{A}, \mathscr{R})$ of a knot $K$ gives rise to a group cycle represented by a cyclic branched covering along $K$ and the representation induced from the arc coloring $\mathscr{A}$.

9A. Let $X$ be a quandle. Let $D$ be a diagram of a knot $K$. For a shadow coloring $\mathscr{Y}=(\mathscr{A}, \mathscr{R})$ of $D$ whose arcs and regions are colored by $X$, define $\mathscr{A} * a$ and $\mathscr{R} * a$ for $a \in X$ by

$$
(\mathscr{A} * a)(x)=\mathscr{A}(x) * a, \quad(\mathscr{R} * a)(r)=\mathscr{R}(r) * a \quad \text { (for any arc } x \text { and region } r) .
$$

By the axiom (Q3), $\mathscr{S} * a=(\mathscr{A} * a, \mathscr{R} * a)$ is also a shadow coloring.

In the following, we assume that $X=\operatorname{Conj}(h)$ for some group $G$ and that $h$ is in $G$ and satisfies Assumption 8.6. As in the previous section, let $s: \operatorname{Conj}(h) \cong$ $Z(h) \backslash G \rightarrow G$ be a section satisfying $s(a * b)=s(a) \widetilde{*} s(b)$ for $a, b \in X$. Let

$$
\iota: C_{n}^{\Delta}(X) \rightarrow C_{n}(G ; \mathbb{Z}):\left(a_{0}, \ldots, a_{n}\right) \mapsto\left(s\left(a_{0}\right), \ldots, s\left(a_{n}\right)\right) .
$$

Composing with $\varphi: C_{n}^{Q}(X ; \mathbb{Z}[X]) \rightarrow C_{n+1}^{\Delta}(X)$, we have a map

$$
C_{2}^{Q}(X ; \mathbb{Z}[X]) \stackrel{\varphi}{\rightarrow} C_{3}^{\Delta}(X) \stackrel{\iota}{\rightarrow} C_{3}(G ; \mathbb{Z}) .
$$

For a shadow coloring $\mathscr{Y}, \iota \varphi(C(\mathscr{Y}))$ is not a cycle of $C_{3}(G ; \mathbb{Z})$ in general. But we can show:

Theorem 9.1. Let $\mathscr{Y}=(\mathscr{A}, \mathscr{R})$ be a shadow coloring of a diagram $D$ of a knot $K$ by $\operatorname{Conj}(h)$. Let $a \in \operatorname{Conj}(h)$ be the color of an arc of $D$. If $h^{l}=1$, then

(9-1) $\iota \varphi(C(\mathscr{S}))+\iota \varphi(C(\mathscr{S} * a))+\iota \varphi\left(C\left(\mathscr{S} * a^{2}\right)\right)+\cdots+\iota \varphi\left(C\left(\mathscr{S} * a^{l-1}\right)\right) \in C_{3}(G ; \mathbb{Z})$

is a group cycle represented by the l-fold cyclic branched covering $\widehat{C}_{l}$ along the knot $K$ and the representation $\pi_{1}\left(\widehat{C}_{l}\right) \rightarrow G$ induced from the arc coloring $\mathscr{A}$.

Proof. Let $x_{1}, \ldots, x_{n}$ be the arcs of the diagram $D$ such that $a=\mathscr{A}\left(x_{1}\right)$. We denote the color $\mathscr{A}\left(x_{i}\right)$ by $a_{i}\left(a_{1}=a\right)$. The arc coloring $\mathscr{A}$ induces a representation $\rho: \pi_{1}\left(S^{3}-K\right) \rightarrow G$. Using $s: Z(h) \backslash G \rightarrow G, \rho$ is given by

$$
\rho\left(x_{i}\right)=s\left(a_{i}\right)^{-1} h s\left(a_{i}\right) \in \operatorname{Conj}(h) \subset G .
$$

We have

$$
\begin{aligned}
s\left(b * a_{i}\right) & =h^{-1} s(b) s\left(a_{i}\right)^{-1} h s\left(a_{i}\right)=h^{-1} s(b) \rho\left(x_{i}\right) \in G, \\
s\left(b *^{-1} a_{i}\right) & =h s(b) s\left(a_{i}\right)^{-1} h^{-1} s\left(a_{i}\right)=h s(b) \rho\left(x_{i}\right)^{-1} \in G,
\end{aligned}
$$




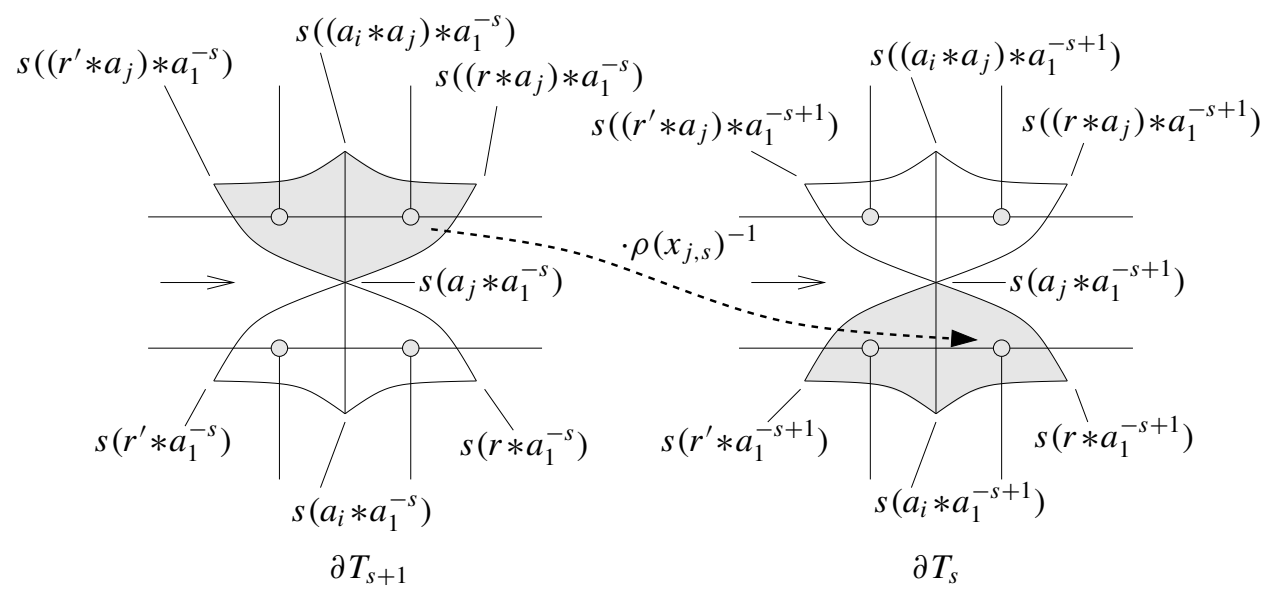

Figure 14. A labeling at a crossing. $\left(r^{\prime}=r *^{ \pm 1} a_{i}\right.$.)

for any $b \in \operatorname{Conj}(h)$. So we have

$$
\begin{gathered}
s\left(\left(b * a_{i}\right) * a_{1}^{-s}\right)=h^{s-1} s(b) \rho\left(x_{i}\right) \rho\left(x_{1}\right)^{-s}, \\
s\left(b * a_{1}^{-s+1}\right)=h^{s-1} s(b) \rho\left(x_{1}\right)^{-s+1} .
\end{gathered}
$$

Since

$$
\rho\left(x_{i, s}\right)=\rho\left(x_{1}^{s-1} x_{i} x_{1}^{-s}\right)=\rho\left(x_{1}\right)^{s-1} \rho\left(x_{i}\right) \rho\left(x_{1}\right)^{-s}
$$

by (4-1), we have

$$
s\left(b * a_{1}^{-s+1}\right)=s\left(\left(b * a_{i}\right) * a_{1}^{-s}\right) \rho\left(x_{i, s}\right)^{-1} .
$$

Let $T_{s}(s=0,1, \ldots l-1)$ be copies of the triangulation of a 3-ball with a vertex ordering on each tetrahedron constructed in Section 4 (Figures 5 and 7). Then we define a labeling of vertices of $T_{s}$ by $\iota \varphi\left(C\left(\mathscr{Y} * a_{1}^{-s+1}\right)\right)$ (Figure 14). The vertices of the face ${F^{\prime}}_{j, s+1}^{-} \subset T_{s+1}$ are labeled by

$$
\left(s\left(\left(r * a_{j}\right) * a_{1}^{-s}\right), \quad s\left(\left(a_{i} * a_{j}\right) * a_{1}^{-s}\right), \quad s\left(a_{j} * a_{1}^{-s}\right)\right),
$$

and the vertices of the face $F_{j, s}^{\prime+} \subset T_{s}$ are labeled by

$$
\left(s\left(r * a_{1}^{-s+1}\right), \quad s\left(a_{i} * a_{1}^{-s+1}\right), \quad s\left(a_{j} * a_{1}^{-s+1}\right)\right),
$$

where $r$ is the color of the region as indicated in Figure 14. By (9-2), the labelings of the face ${F^{\prime}}_{j, s+1}^{-}$and ${F^{\prime}}_{j, s}^{+}$are related by

$$
\begin{aligned}
\left(s\left(r * a_{1}^{-s+1}\right),\right. & \left.s\left(a_{i} * a_{1}^{-s+1}\right), \quad s\left(a_{j} * a_{1}^{-s+1}\right)\right) \\
= & \left(s\left(\left(r * a_{j}\right) * a_{1}^{-s}\right), \quad s\left(\left(a_{i} * a_{j}\right) * a_{1}^{-s}\right), \quad s\left(a_{j} * a_{1}^{-s}\right)\right) \rho\left(x_{j, s}\right)^{-1} .
\end{aligned}
$$


Therefore this gives a $G$-valued 1-cocycle on $\widehat{T}$ as constructed in Section 4 . Thus the chain given by (9-1) is a cycle represented by the cyclic branched cover $\widehat{C}_{l}$ and the representation induced from the arc coloring $\mathscr{A}$.

9B. We end this section by comparing the shadow cocycle invariant of the $(2, p)$ torus knot ( $p$ : odd) for the 3-cocycle obtained in Section 7 and the Dijkgraaf-Witten invariant of the lens space $L(p, 1)$.

Let $G$ be a finite group and $\alpha$ be a cocycle of $H^{3}(G ; A)$, where $A$ is an abelian group. Dijkgraaf and Witten defined an invariant of closed oriented 3-manifolds for each $\alpha$. For an oriented closed manifold $M$, it is defined by

$$
\sum_{\rho: \pi_{1}(M) \rightarrow G}\left\langle\rho^{*} \alpha,[M]\right\rangle \in \mathbb{Z}[A],
$$

where $\rho^{*} \alpha$ is the pull-back of $\alpha$ by the classifying map $M \rightarrow B G$ corresponding to $\rho$. Since $L(p, 1)$ is a double branched cover along the $(2, p)$-torus knot, the cycle obtained from a shadow coloring by $R_{p}$ gives rise to a group 3-cycle of $D_{2 p}$ represented by $L(p, 1)$ by Theorem 9.1. Since every representation of $\pi_{1}(L(p, 1)) \cong \mathbb{Z} / p$ into $D_{2 p}$ reduces to a representation into $\mathbb{Z} / p$, it is natural to ask whether the shadow cocycle invariant for our quandle 3-cocycle coincides with the Dijkgraaf-Witten invariant for $b_{1} b_{2} \in H^{3}(\mathbb{Z} / p ; \mathbb{Z} / p)$. As we remarked in the introduction, these invariants coincide up to some constant by the result of Hatakenaka and Nosaka [2012] when $p$ is prime.

Let $\mathscr{Y}$ be the shadow coloring indicated in Figure 12. Since the homology class $[C(\mathscr{Y})]$ does not change under the action of $R_{p}$ on the shadow coloring $\mathscr{Y}$ (see Lemma 4.5 of [Inoue and Kabaya 2010]), we assume that $x=0$. As computed in Section 7B, the evaluation of the $C(\mathscr{S})$ at the 3 -cocycle derived from $b_{1} b_{2}$ is equal to $-4 y^{2} \bmod p$. Therefore the shadow cocycle invariant is

$$
p \sum_{y=0}^{p-1} t^{-4 y^{2}}=p \sum_{y=0}^{p-1} t^{-y^{2}} \in \mathbb{Z}[t] /\left(t^{p}-1\right) \cong \mathbb{Z}[\mathbb{Z} / p] .
$$

We compute the Dijkgraaf-Witten invariant of the lens space $L(p, q)$ for the group 3-cocycle $b_{1} b_{2} \in H^{3}(\mathbb{Z} / p ; \mathbb{Z} / p)$. Although this was computed in [Murakami et al. 1992], we give a proof based on a triangulation. We represent $L(p, q)$ by an ordered 3-cycle and give a labeling of 1-simplices as indicated in Figure 15. Here the triple $a, b$ and $c$ must satisfy $a^{p}=1$ and $b=a^{q} b c$. Using multiplicative notation, the evaluation of $b_{1} b_{2}$ on this cycle is

$$
\sum_{i=0}^{p-1} b_{1} b_{2}([a|i a+b|-q a])=\sum_{i=0}^{p-1} a \cdot b_{2}(i a+b,-q a)=-q a^{2} .
$$



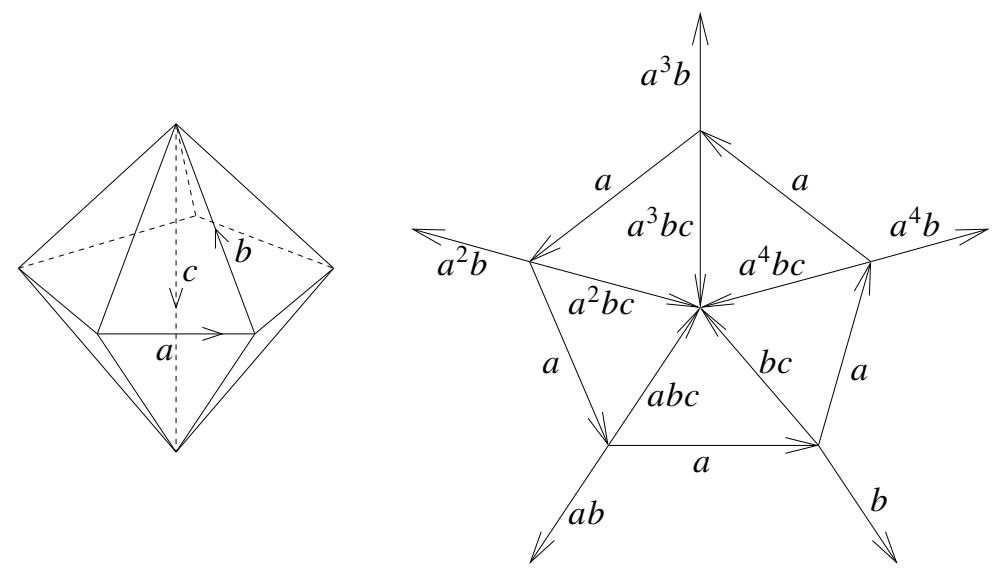

Figure 15. A triangulation of $L(5, q)$ and a labeling where $a^{5}=1$ and $b=a^{q} b c$.

The second equality follows from the fact that $i a+b$ runs over $\mathbb{Z} / p$ and from (7-1) when $a$ is a unit, and also when $a$ is not a unit by a similar argument. Therefore the Dijkgraaf-Witten invariant of $L(p, q)$ is equal to

$$
\sum_{a=0}^{p-1} t^{-q a^{2}} \in \mathbb{Z}[t] /\left(t^{p}-1\right) \cong \mathbb{Z}[\mathbb{Z} / p] .
$$

Therefore this coincides with the shadow cocycle invariant up to a constant.

\section{Acknowledgements}

The author thanks Takefumi Nosaka for useful discussions. He also thanks the referees for helpful comments for improving the exposition.

\section{References}

[Benson 1991] D. J. Benson, Representations and cohomology, I: Basic representation theory of finite groups and associative algebras, Cambridge Studies in Advanced Mathematics 30, Cambridge University Press, 1991. MR 92m:20005 Zbl 0718.20001

[Brown 1982] K. S. Brown, Cohomology of groups, Graduate Texts in Mathematics 87, Springer, New York, 1982. MR 83k:20002 Zbl 0584.20036

[Carter et al. 2003] J. S. Carter, D. Jelsovsky, S. Kamada, L. Langford, and M. Saito, "Quandle cohomology and state-sum invariants of knotted curves and surfaces", Trans. Amer. Math. Soc. 355:10 (2003), 3947-3989. MR 2005b:57048 Zbl 1028.57003

[Clauwens 2011] F. Clauwens, "The algebra of rack and quandle cohomology", J. Knot Theory Ramifications 20:11 (2011), 1487-1535. MR 2854230 Zbl 05998125 
[Eisermann 2003] M. Eisermann, "Homological characterization of the unknot", J. Pure Appl. Algebra 177:2 (2003), 131-157. MR 2003j:57009 Zbl 1013.57002

[Eisermann 2007] M. Eisermann, "Knot colouring polynomials", Pacific J. Math. 231:2 (2007), 305-336. MR 2008j:57014 Zbl 1152.57010

[Etingof and Graña 2003] P. Etingof and M. Graña, "On rack cohomology", J. Pure Appl. Algebra 177:1 (2003), 49-59. MR 2004e:55006 Zbl 1054.16028

[Fenn et al. 1995] R. Fenn, C. Rourke, and B. Sanderson, "Trunks and classifying spaces", Appl. Categ. Structures 3:4 (1995), 321-356. MR 96i:57023 Zbl 0853.55021

[Hatakenaka and Nosaka 2012] E. Hatakenaka and T. Nosaka, "Some topological aspects of 4-fold symmetric quandle invariants of 3-manifolds", Int. J. Math. 23:7 (2012), Art. ID 1250064.

[Inoue and Kabaya 2010] A. Inoue and Y. Kabaya, "Quandle homology and complex volume", preprint, 2010. arXiv 1012.2923v1

[Joyce 1982] D. Joyce, "A classifying invariant of knots, the knot quandle”, J. Pure Appl. Algebra 23:1 (1982), 37-65. MR 83m:57007 Zbl 0474.57003

[Kamada 2007] S. Kamada, "Quandles with good involutions, their homologies and knot invariants", pp. 101-108 in Intelligence of low dimensional topology 2006 (Hiroshima, 2006), edited by J. S. Carter et al., Ser. Knots Everything 40, World Sci., Hackensack, NJ, 2007. MR 2009a:57042 Zbl 1145.57008

[Mochizuki 2003] T. Mochizuki, "Some calculations of cohomology groups of finite Alexander quandles”, J. Pure Appl. Algebra 179:3 (2003), 287-330. MR 2004b:55013 Zbl 1036.18009

[Murakami et al. 1992] H. Murakami, T. Ohtsuki, and M. Okada, "Invariants of three-manifolds derived from linking matrices of framed links", Osaka J. Math. 29:3 (1992), 545-572. MR 93h:57013 Zbl 0776.57009

[Neumann 2004] W. D. Neumann, "Extended Bloch group and the Cheeger-Chern-Simons class", Geom. Topol. 8 (2004), 413-474. MR 2005e:57042 Zbl 1053.57010

[Nosaka 2009] T. Nosaka, "On quandle homology groups of Alexander quandles of prime order", preprint, 2009, available at http://www.kurims.kyoto-u.ac.jp/preprint/file/RIMS1680.pdf. To appear in Trans. Amer. Math. Soc.

[Nosaka 2010] T. Nosaka, "Quandle homotopy invariants of knotted surfaces", preprint, 2010. arXiv 1011.6035

[Rolfsen 1976] D. Rolfsen, Knots and links, Mathematics Lecture Series 7, Publish or Perish, Berkeley, CA, 1976. MR 58 \#24236 Zbl 0339.55004

[Weeks 2005] J. Weeks, "Computation of hyperbolic structures in knot theory", pp. 461-480 in Handbook of knot theory, edited by W. Menasco and M. Thistlethwaite, Elsevier, Amsterdam, 2005. MR 2006k:57027 Zbl 1096.57015

Received November 9, 2011. Revised April 13, 2012.

\section{YUICHI KABAYA}

DEPARTMENT OF MATHEMATICS

OSAKA UNIVERSITY

TOYONAKA, OSAKA 560-0043

JAPAN

y-kabaya@cr.math.sci.osaka-u.ac.jp 


\title{
PACIFIC JOURNAL OF MATHEMATICS
}

\author{
http://pacificmath.org \\ Founded in 1951 by \\ E. F. Beckenbach (1906-1982) and F. Wolf (1904-1989)
}

\section{EDITORS}

V. S. Varadarajan (Managing Editor)

Department of Mathematics

University of California

Los Angeles, CA 90095-1555

pacific@math.ucla.edu

Vyjayanthi Chari

Department of Mathematics

University of California

Riverside, CA 92521-0135

chari@math.ucr.edu

\section{Robert Finn}

Department of Mathematics Stanford University

Stanford, CA 94305-2125

finn@math.stanford.edu

Kefeng Liu

Department of Mathematics

University of California

Los Angeles, CA 90095-1555

liu@math.ucla.edu
Darren Long

Department of Mathematics

University of California

Santa Barbara, CA 93106-3080

long@math.ucsb.edu

Jiang-Hua Lu

Department of Mathematics

The University of Hong Kong

Pokfulam Rd., Hong Kong jhlu@maths.hku.hk

Alexander Merkurjev

Department of Mathematics

University of California

Los Angeles, CA 90095-1555

merkurev@math.ucla.edu
Sorin Popa

Department of Mathematics University of California

Los Angeles, CA 90095-1555 popa@math.ucla.edu

Jie Qing

Department of Mathematics

University of California

Santa Cruz, CA 95064

qing@cats.ucsc.edu

Jonathan Rogawski

Department of Mathematics

University of California

Los Angeles, CA 90095-1555

jonr@math.ucla.edu

\section{PRODUCTION}

pacific@math.berkeley.edu

\section{SUPPORTING INSTITUTIONS}

ACADEMIA SINICA, TAIPEI

CALIFORNIA INST. OF TECHNOLOGY INST. DE MATEMÁTICA PURA E APLICADA KEIO UNIVERSITY

MATH. SCIENCES RESEARCH INSTITUTE NEW MEXICO STATE UNIV.

OREGON STATE UNIV.

\author{
STANFORD UNIVERSITY \\ UNIV. OF BRITISH COLUMBIA \\ UNIV. OF CALIFORNIA, BERKELEY \\ UNIV. OF CALIFORNIA, DAVIS \\ UNIV. OF CALIFORNIA, LOS ANGELES \\ UNIV. OF CALIFORNIA, RIVERSIDE \\ UNIV. OF CALIFORNIA, SAN DIEGO \\ UNIV. OF CALIF., SANTA BARBARA
}

\author{
UNIV. OF CALIF., SANTA CRUZ \\ UNIV. OF MONTANA \\ UNIV. OF OREGON \\ UNIV. OF SOUTHERN CALIFORNIA \\ UNIV. OF UTAH \\ UNIV. OF WASHINGTON \\ WASHINGTON STATE UNIVERSITY
}

These supporting institutions contribute to the cost of publication of this Journal, but they are not owners or publishers and have no responsibility for its contents or policies.

See inside back cover or pacificmath.org for submission instructions.

The subscription price for 2012 is US \$420/year for the electronic version, and \$485/year for print and electronic.

Subscriptions, requests for back issues from the last three years and changes of subscribers address should be sent to Pacific Journal of Mathematics, P.O. Box 4163, Berkeley, CA 94704-0163, U.S.A. Prior back issues are obtainable from Periodicals Service Company, 11 Main Street, Germantown, NY 12526-5635. The Pacific Journal of Mathematics is indexed by Mathematical Reviews, Zentralblatt MATH, PASCAL CNRS Index, Referativnyi Zhurnal, Current Mathematical Publications and the Science Citation Index.

The Pacific Journal of Mathematics (ISSN 0030-8730) at the University of California, c/o Department of Mathematics, 969 Evans Hall, Berkeley, CA 94720-3840, is published monthly except July and August. Periodical rate postage paid at Berkeley, CA 94704, and additional mailing offices. POSTMASTER: send address changes to Pacific Journal of Mathematics, P.O. Box 4163, Berkeley, CA 94704-0163.

PJM peer review and production are managed by EditFLOW ${ }^{\mathrm{TM}}$ from Mathematical Sciences Publishers.

PUBLISHED BY PACIFIC JOURNAL OF MATHEMATICS

at the University of California, Berkeley 94720-3840

A NON-PROFIT CORPORATION

Typeset in LATEX

Copyright $(02012$ by Pacific Journal of Mathematics 


\section{PACIFIC JOURNAL OF MATHEMATICS}

Volume $259 \quad$ No. $2 \quad$ October 2012

Flag subdivisions and $\gamma$-vectors

257

Christos A. ATHANASIAdis

Rays and souls in von Mangoldt planes

279

IGOR BELEGRADEK, ERIC CHOI and NOBUHIRO INNAMI

Isoperimetric surfaces with boundary, II

ABRAHAM FRANDSEN, DONALD SAMPSON and NEIL

STEINBURG

Cyclic branched coverings of knots and quandle homology

YUICHI KABAYA

On a class of semihereditary crossed-product orders

JOHN S. KAUTA

An explicit formula for spherical curves with constant torsion

DEMETRE KAZARAS and IVAN STERLING

Comparing seminorms on homology

JEAN-FranÇOIS LAFONT and CHRISTOPHE PITTET

Relatively maximum volume rigidity in Alexandrov geometry

NAN LI and XIAOCHUN RONG

Properness, Cauchy indivisibility and the Weil completion of a group of 421 isometries

Antonios Manoussos and Polychronis Strantzalos

Theta lifts of strongly positive discrete series: the case of $(\tilde{\mathrm{Sp}}(n), O(V)) 445$

IVAN MATIĆ

Tunnel one, fibered links

MATt RATHBUN

Fusion symmetric spaces and subfactors

HANS WENZL 